\title{
Chemical inhibition of pathological reactive astrocytes promotes neural protection
} Benjamin L.L. Clayton ${ }^{1 凹}$, James D. Kristell ${ }^{1}$, Kevin C. Allan ${ }^{1}$, Molly Karl ${ }^{2}$, Eric Garrison ${ }^{2}$, Yuka MaenoHikichi $^{1}$, Annalise M. Sturno ${ }^{1}$, H. Elizabeth Shick ${ }^{1}$, Robert H. Miller ${ }^{2}$, and Paul J. Tesar ${ }^{1 囚}$.

${ }^{1}$ Department of Genetics and Genome Sciences, Case Western Reserve University School of Medicine, Cleveland, OH, USA.

${ }^{2}$ Department of Anatomy and Regenerative Biology, George Washington University School of Medicine, Washington D.C., USA.

$\unrhd_{\text {email: paul.tesar@case.edu; benjamin.clayton@case.edu }}$

\begin{abstract}
Disease, injury, and aging induce reactive astrocyte states with pathological functions ${ }^{1-4}$. In neurodegenerative diseases, inflammatory reactive astrocytes are abundant and contribute to progressive cell loss. Modulating the state or function of these reactive astrocytes thereby represents an attractive therapeutic goal ${ }^{5,6}$. Leveraging a cellular phenotypic screening platform, we show that chemical inhibitors of HDAC3 effectively block pathological astrocyte reactivity. Inhibition of HDAC3 reduces molecular and functional features of reactive astrocytes in vitro including inflammatory gene expression, cytokine secretion, and antigen presentation. Transcriptional and chromatin mapping studies show that HDAC3 inhibition mediates a switch between pro-inflammatory and anti-inflammatory states, which disarms the pathological functions of reactive astrocytes. Systemic administration of a blood-brain barrier penetrant chemical inhibitor of HDAC3, RGFP966, blocks reactive astrocyte formation and promotes axonal protection in vivo. Collectively, these results establish a platform for discovering chemical modulators of reactive astrocyte states, inform the mechanisms controlling astrocyte reactivity, and demonstrate the therapeutic potential of modulating astrocyte reactivity for neurodegenerative diseases.
\end{abstract}

\section{Main}

Astrocytes in the central nervous system (CNS) play important homeostatic roles that include trophic support of neurons, promotion of functional synapse formation, and formation and maintenance of the blood-brain barrier ${ }^{7-9}$. In the context of disease, injury, or normal aging, astrocytes become reactive and can adopt a pathological state that kills neurons and oligodendrocytes $^{6}$. These pathogenic reactive astrocytes are found in neurodegenerative diseases including Alzheimer's disease, Parkinson's disease, Huntington's disease, amyotrophic lateral 
sclerosis, vanishing white matter disease ${ }^{10}$, and multiple sclerosis ${ }^{1,11}$. Thus, there is considerable interest in modulating pathological reactive astrocytes to reduce the progression of these diseases $^{3,6,12}$. Here, we develop an astrocyte discovery platform and leverage the power of highthroughput phenotypic drug screening ${ }^{13}$ to identify modulators of reactive astrocytes. We identify HDAC3 as a druggable target that modulates reactive astrocytes in vitro and in vivo by suppressing pro-inflammatory gene programs while inducing expression of anti-inflammatory genes and genes associated with a beneficial reactive astrocyte state. Using a CNS-penetrant HDAC3 inhibitor, we further show that HDAC3 inhibition decreases the formation of reactive astrocytes and that suppression of pathological reactive astrocytes through HDAC3 inhibition promotes axonal protection in vivo.

\section{An astrocyte discovery platform for high-throughput phenotypic drug screening}

Current astrocyte isolation protocols are limited by scale or confounded by culture conditions containing serum, which irreversibly alters the astrocyte transcriptome and morphology ${ }^{14}$. This challenges their use as cellular platforms for high-throughput drug screening. To overcome this, we developed an approach to isolate and culture large numbers (hundreds of millions) of resting mouse cortical astrocytes that exhibit a prototypical stellate morphology and express canonical astrocyte marker proteins including GFAP, AQP4, GLT-1, and ALDH1L1 (Extended Data Fig. 1a,b). Using single-cell RNA sequencing and in situ hybridization, we demonstrated the high purity of these cultures and confirmed that the astrocytes become reactive in response to microglial-derived cytokines tumor necrosis factor (TNF), interleukin 1 alpha (IL1 $\alpha$ ), and complement component 1q (C1q) ${ }^{1}$ (Fig. 1a-c). Comparison to publicly available single-cell RNA sequencing data from systemic lipopolysaccharide (LPS) driven neuroinflammation in mice demonstrated that these reactive astrocytes increase expression of genes that define an in vivo neuroinflammatory specific astrocyte state (Extended Data Fig. 1c), which is also found in mouse models of Alzheimer's disease, multiple sclerosis, and stab wound injury ${ }^{3}$. Additional comparison to astrocyte transcriptional signatures from single-nuclei analysis of Alzheimer's disease ${ }^{15}$, Huntington's disease ${ }^{16}$, and Parkinson's disease ${ }^{17}$ patient tissue showed that our reactive astrocytes reflect the pathological reactive astrocyte states found in these human neurodegenerative diseases (Fig. 1d-f). Finally, we confirmed that our reactive astrocyte cultures acquire functions of reactive astrocytes including cross-presentation of antigen on major histocompatibility complex (MHC) 
Class I and increased secretion of proinflammatory cytokines ${ }^{18}$ (Fig. 1g,h). Together, these results show that this culture system generates pure astrocytes that robustly transition to a disease-relevant state.

\section{Phenotypic drug screen identifies small-molecules that suppress pathological reactive astrocytes}

Leveraging our astrocyte discovery platform we used the power of high-throughput phenotypic screening to identify small-molecules that suppress astrocyte reactivity. Resting astrocytes exposed to TNF, IL1 $\alpha$, and C1q were treated with 3115 small molecules in a 384-well plate format and, after 24 hours, their transition to reactive astrocytes was measured with the marker protein guanylate-binding protein 2 (GBP2), which is specifically upregulated in both mouse and human reactive astrocytes in a pathological state ${ }^{1,19}$. The assay displayed consistently high signal-tobackground ratio across all screening plates, demonstrating its suitability as a phenotypic endpoint (Extended Data Fig. 2a-c). Our primary screen successfully identified hit compounds that decreased the percentage of GBP2-positive astrocytes by $\geq 90 \%$ compared to cultures treated with DMSO vehicle (Fig. 1i). Of those primary hits, 29 small molecules were validated to modulate reactive astrocytes over at least 3 concentrations and decrease the expression of proteasome $20 \mathrm{~S}$ subunit beta $8(P s m b 8)$ as a secondary marker of pathological reactive astrocytes (Extended Data Fig. 2d,e). Validated hits covered a broad range of compound classes but were highly enriched for histone deacetylase (HDAC) inhibitors that accounted for 48\% (14/29) of the hits (Fig. 1j and 2a). There are 11 HDAC isozymes and many chemical HDAC inhibitors are non-specific. Only HDAC3 was a shared target of all 14 HDAC inhibitors identified in our primary screen, including the only isozyme-specific chemical, RGFP966 (Extended Data Fig. 3a). RGFP966 is an HDAC3 specific inhibitor with no reported inhibition of other HDAC isozymes at up to $15 \mathrm{uM}^{20}$. This led us to hypothesize that HDAC3 was the HDAC inhibitor target responsible for modulating pathological reactive astrocytes.

\section{HDAC3 regulates pathological reactive astrocyte gene expression and function}

Using an additional HDAC3 specific inhibitor, $\mathrm{T} 247^{21}$, and genetic ablation of $H d a c 3^{22,23}$, we pharmacologically and genetically confirmed that HDAC3 inhibition modulates pathological reactive astrocytes (Extended Data Fig. 3b-f). Inhibition of HDAC3 in human astrocyte cultures 
also suppressed astrocyte reactivity showing that the effects of HDAC3 inhibition are conserved across species (Extended Data Fig. 3g). RNA sequencing also confirmed that RGFP966 treatment significantly downregulated expression of multiple defining genes of pathological reactive astrocytes, including C3 and Serpingl, showing that the effects were global and not limited to decreasing Gbp2 (Fig. 2b). Pharmacological inhibition of HDAC3 by RGFP966 also suppressed the MHC Class I antigen cross-presentation and inflammatory cytokine secretion functions of pathological reactive astrocytes (Fig. 2c-e). These data show that HDAC3 inhibition reduces pathological reactive astrocyte gene expression and function.

To understand how HDAC3 inhibitors regulate pathological reactive astrocytes, we sought to define the global chromatin and gene expression changes that occur as astrocytes transition to a pathological reactive state (Fig. 2f). H3K27ac ChIP-seq ${ }^{24,25}$ revealed substantial chromatin remodeling in pathological reactive astrocytes, including changes in super-enhancers that are regions of densely-packed active enhancers that control gene expression and cell state ${ }^{26}$. In pathological reactive astrocytes, nearly two-thirds of the super-enhancers were distinct from resting astrocytes demonstrating a substantial shift in the chromatin state (Fig. 2g). Expression of genes associated with gained super-enhancers in reactive astrocytes were significantly upregulated and included cytokines and chemokines involved in innate and adaptive immunity (Cxcll, Ccl5, Cxcl12), while expression of genes associated with shared super-enhancers did not change and included astrocyte defining lineage markers (Nfia. Sox 9, Fgfrl, and Aqp4 $4^{27}$ (Fig. 2h-j). HDAC3 inhibition by RGFP966 significantly decreased expression of gained super-enhancer genes but did not alter shared or lost super enhancer gene expression in reactive astrocytes (Fig. 2k). These data show that HDAC3 inhibition suppresses the transcripts induced in pathological reactive astrocytes by their dramatically altered chromatin landscape.

To uncover the regulators of astrocyte reactivity, we performed ATAC-seq and mined for motifs under open chromatin regions within gained super-enhancers in pathological reactive astrocytes. This analysis identified the RelA/p65 subunit of NF- $\mathrm{kB}$ as the top putative driver shaping the reactive astrocyte chromatin landscape (Extended Data Fig. 4a). Gene ontology analysis also highlighted NF- $\mathrm{KB}$ signaling as an enriched pathway in genes targeted by pathological reactive astrocyte gained super-enhancers (Extended Data Fig. 4b). Consistent with these data, we found a 
significant increase in nuclear RelA/p65 protein in reactive astrocytes that was abrogated by HDAC3 inhibition with RGFP966 (Extended Data Fig. 4c-e). Strikingly, we found that 82.75\% (24/29) of the validated hits from our primary screen, including RGFP966, significantly inhibited RelA/p65 transcriptional activity in an orthogonal reporter assay (Extended Data Fig. 4f,g). Collectively, these data highlight a critical role of RelA/p65 in regulating pathological astrocyte reactivity and show that multiple molecular nodes, including HDAC3, can be targeted pharmacologically to impede RelA/p65 and modulate pathological reactive astrocytes.

\section{HDAC3 inhibition mediates a switch between pro- and anti-inflammatory astrocyte programs}

To explore the role of RelA/p65 as a driver of reactive astrocytes in a pathological state, we performed RelA/p65 ChIP-seq in resting and pathological reactive astrocytes. We found that RelA/p65 DNA binding increased significantly as astrocytes become reactive (Fig. 3a), and that the majority of RelA/p65 direct target genes (genes with a RelA/p65 peak within $5 \mathrm{~Kb}$ up- or downstream of the transcription start site) had increased expression $(60.43 \%, 113 / 187)$ (Fig. 3b). These included inflammatory cytokines and chemokines ( $\mathrm{Cxcl10}$ and $\mathrm{Ccl}$ ), genes involved in MHC Class I antigen presentation ( $H 2-D 1, B 2 m$, and $H 2-K 1)$, and genes that define pathological reactive astrocytes (C3 and Gbp2), (Fig. 3b). However, direct targets of RelA/p65 accounted for a small proportion (113/1839 upregulated transcripts, 9/1789 downregulated transcripts) of the overall gene expression changes in pathological reactive astrocytes, indicating that multiple regulatory programs may be mediating the generation of reactive astrocytes.

Non-RelA/p65 direct target genes with increased expression in pathological reactive astrocytes also included inflammatory cytokines and chemokines $(\mathrm{Cxcl5}, \mathrm{Ccl}$, and $\mathrm{Il} 6)$, transcription factors involved in acute inflammation (Cebpb and Cebpd), the immunoproteasome (Psmb8, Psmb9, and Psmb10), interferon-induced genes (Ifi47, Irf7, Iigbp1, Iigbp7, and Ifitm3), and genes involved in lipid metabolism and transport (Apoj/Clu and Elovll) (Fig. 3c). Gene ontology analysis of RelA/p65 and non-RelA/p65 direct target genes identified similar but not identical biological processes regulated by the two gene programs (Fig. 3d). However, transcription factor motifs enriched in the promoters of direct RelA/p65 and non-RelA/p65 direct target genes were distinct suggesting they are controlled by different mechanisms (Fig. 3e,f). HDAC3 inhibition by 
RGFP966 decreased expression of both RelA/p65 and non-RelA/p65 direct target genes in pathological reactive astrocytes (Fig. 3g). These included RelA/p65 direct target MHC Class I genes involved in the antigen presentation function blocked by RGFP966, and non-RelA/p65 direct target genes associated with the metabolism and transport of very-long chain fatty acids that were recently shown to partially mediate pathological reactive astrocyte toxicity to neurons and oligodendrocytes $^{6}$ (Fig. 3h-j). These data show that HDAC3 inhibition broadly suppresses the multiple transcriptional programs that regulate pathological reactive astrocyte formation.

While most transcripts changed by RGF9666 in reactive astrocytes were decreased, nearly $40 \%$ of all RGFP966 changed transcripts (539/1380) were increased. Transcripts increased in reactive astrocytes by RGFP966 treatment included numerous genes associated with a beneficial reactive

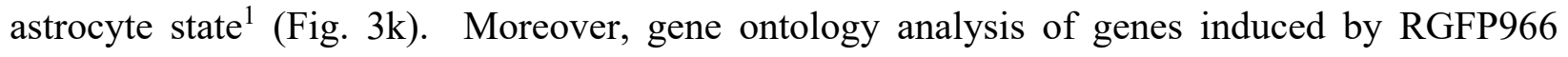
treatment was enriched for terms associated with response to oxidative stress, glutathione metabolism, and KEAP1-NRF2 signaling (Fig. 31). NRF2 activation in astrocytes has been shown to protect against neurodegeneration and negatively regulate inflammation ${ }^{3,28}$. Importantly, we found that HDAC3 inhibition with RGFP966 activated NRF2 signaling. Cytoprotective Nrf2 target genes $^{29}$ including those involved in glutathione production, antioxidant systems, NADPH regeneration, and heme and iron metabolism were significantly induced by RGFP966 treatment (Fig. 3m). These findings show that HDAC3 inhibition not only mitigates proinflammatory target gene expression, but also promotes expression of genes associated with an anti-inflammatory beneficial astrocyte state. This suggests that HDAC3 acts as a molecular switch between reactive astrocyte states where HDAC3 inhibition promotes an anti-inflammatory and protective astrocyte phenotype.

\section{Pharmacological inhibition of HDAC3 promotes neuroprotection in vivo}

To examine the therapeutic effect of modulating reactive astrocytes, we tested whether chemical HDAC3 inhibition could reduce pathological reactive astrocytes in vivo. With daily intraperitoneal (i.p.) dosing of 10mg/kg, RGFP966 crossed the blood-brain barrier and reached a concentration in brain tissue near in vitro IC50 levels required to inhibit astrocyte reactivity (Extended Data Fig. 5a). RGFP966 increased gross acetylated histone 4 ( $\mathrm{AcH} 4)$ brain levels in vivo demonstrating a pharmacodynamic effect to inhibit the histone deacetylase activity of HDAC3 (Extended Data Fig. 
5b-d). We used the well-established model of systemic LPS-induced neuroinflammation ${ }^{30,31}$ to test whether HDAC3 inhibition with RGFP966 could modulate pathological reactive astrocytes in vivo. Compared to vehicle, treatment with $10 \mathrm{mg} / \mathrm{kg}$ RGFP966 successfully decreased the formation of Gbp2-positive pathological reactive astrocytes in LPS-exposed mice (Fig. 4a-c). Importantly, RGFP966 treatment had no effect on general astrocyte reactivity as measured with GFAP and had no effect on microglial activation measured with IBA-1(Extended Data Fig. 5e-k). This shows that HDAC3 inhibition with RGFP966 can specifically decrease the formation of the pathological reactive astrocyte state in vivo.

Finally, we examined whether decreasing pathological reactive astrocyte formation by pharmacologically inhibiting HDAC3 could promote neural protection. To do this, we used a toxin-based model of CNS tissue damage where lysolecithin (lysophosphatidylcholine; LPC) is injected into the dorsal column of the spinal cord, leading to myelinating oligodendrocyte loss, astrogliosis, and both acute and continued axonal degeneration ${ }^{32,33}$. We first confirmed that LPCinduced injury leads to the formation of $C 3$-positive pathological reactive astrocytes, that RGFP966 significantly decreased the formation of these C3-positive pathological reactive astrocytes, and that RGFP966 had no effect on general microgliosis or astrogliosis in this injury model (Fig. 4d,e; Extended Data Fig. 6a-d). Using electron microscopy to assess tissue pathology, we compared spinal cord sections from RGFP966-treated versus vehicle-treated LPC mice and found that HDAC3 inhibition with RGFP966 promoted axonal protection (Fig. 4f). At 12 days post-LPC injury, RGFP966 treatment significantly increased the density of total axons and the density of myelinated axons (Fig. 4g,h). These data show that pathological reactive astrocytes play a role in the loss of both unmyelinated and myelinated axons in the LPC injury model and that pharmacological inhibition of HDAC3 suppressed pathological reactive astrocytes and promoted axonal protection following in vivo tissue damage.

\section{Conclusions}

Here we show the development of an astrocyte discovery platform to identify modulators of reactive astrocytes. A phenotypic chemical screen identified HDAC3 as a functional regulator of a pathological reactive astrocyte state change. We demonstrate that pathological reactive astrocytes represent a cell state change that involves global reorganization of the chromatin 
landscape driven in part by RelA/p65. HDAC3 inhibition in reactive astrocytes suppresses RelA/p65 and non-RelA/p65 proinflammatory gene programs, while increasing expression of antiinflammatory and beneficial reactive astrocyte associated genes. We further show that inhibition of HDAC3 decreases the formation of pathological reactive astrocytes in an in vivo model of neuroinflammation. These findings support the role of HDAC3 as a molecular switch to control astrocyte polarization between pathological or beneficial cell states and suggest that therapeutics targeting HDAC3 in neurodegenerative disease may have the dual benefit of blocking pathological reactive astrocyte formation while promoting the formation of beneficial reactive astrocytes to protect neurons and promote repair. We support this by showing in a model of toxin-induced CNS damage that pharmacological inhibition of HDAC3 decreases the formation of pathological reactive astrocytes and promotes neural protection. These findings provide a foundation for identification of additional pathological reactive astrocyte modulating chemicals, specify a deeper understanding of the molecular regulators of pathological reactive astrocyte gene expression, and support the development of reactive astrocyte-targeted therapies for neurodegenerative diseases. 


\section{Methods}

\section{Lipopolysaccharide (LPS) model of neuroinflammation}

C57BL/6N mice were purchased from Charles River (Wilmington, MA). Male and female mice at 7 weeks of age were injected i.p. with either RGFP966 vehicle (30\% hydroxypropyl- $\beta$ cyclodextrin, $0.1 \mathrm{M}$ sodium acetate, and 10\% DMSO) or 10mg/kg RGFP966 daily for 11 days. After which mice were injected i.p. daily for two days with either LPS vehicle (saline) plus RGFP966 vehicle, 5mg/kg LPS plus RGFP966 vehicle, or 5mg/kg LPS plus 10mg/kg RGFP966. Animals were then sacrificed and processed for immunohistochemistry.

\section{Lysolecithin (LPC) injection model of focal tissue damage}

Focal tissue damage in the spinal cord was induced by the injection of 1\% LPC solution. 10-12week-old C57BL/6 female mice were anesthetized using isoflurane and a T10 laminectomy was performed. $1 \mu \mathrm{L}$ of $1 \% \mathrm{LPC}$ was infused into the dorsal column at a rate of $15 \mathrm{~mL} /$ hour. The animals were euthanized at day twelve after the laminectomy ( $n=3-4$ per group). Animals received either RGFP966 vehicle or 10mg/kg RGFP966 daily by i.p. injections that began 1 day prior to LPC injection and ended 11 days after LPC injection. Experiments were terminated 12 days after LPC injection and tissue was then processed for immunohistochemistry or electron microscopy.

\section{Isolation and generation of primary astrocytes}

Timed-pregnant C57BL/6N mice were purchased from Charles River (Wilmington, MA). Brains from mice of the C57BL/6N strain were extracted at postnatal day 2 (P2). A gross dissection of these brains was performed to isolate the cortices which were then dissociated according to 
manufacturer's instructions using the Miltenyi Tumor Dissociation Kit (Miltenyi, 130-095-929). After dissociation, cells were plated in flasks coated with poly-L-ornithine (Sigma, P3655) and laminin (Sigma, L2020). Cells were cultured for 24 hours in media consisting of Dulbecco's Modified Eagle Medium/Nutrient Mixture F-12 (DMEM/F-12; ThermoFisher 11320033), N-2 MAX Supplement (ThermoFisher, 17502048), B-27 Supplement (ThermoFisher, 17504044), GlutaMAX Supplement (ThermoFisher, 35050079), Penicillin-Streptomycin (ThermoFisher, 15070063), and 20ng/mL FGF-2. After 24 hours, the cells were switched to astrocyte enrichment media, comprised of 1:1 DMEM (ThermoFisher, 11960044) and Neurobasal Medium (ThermoFisher, 21103049) supplemented with GlutaMAX (ThermoFisher, 35050061), Sodium Pyruvate (ThermoFisher, 11360070), N-2 MAX Supplement (R\&D, AR009), 5ug/ml N-acetyl cysteine (Sigma, A8199), Penicillin-Streptomycin (ThermoFisher, 15070-063), 5 ng/mL HB-EGF (R\&D Systems, 259-HE-050), 10 ng/mL CNTF (R\&D Systems, 557-NT-010), 10 ng/mL BMP4 (R\&D Systems, 314-BP-050), and $20 \mathrm{ng} / \mathrm{mL}$ FGF2 (R\&D Systems, 233-FB-01M) to proliferate. Media was changed every 48 hours. Once confluent, astrocytes were either cryopreserved or passaged once and then cryopreserved. To conduct a terminal experiment, cryopreserved astrocytes were removed from liquid nitrogen storage and thawed in astrocyte maturation media (1:1 DMEM and Neurobasal Medium with GlutaMAX Supplement, Sodium Pyruvate, N-2 MAX Supplement, N-acetyl cysteine supplemented with $5 \mathrm{ng} / \mathrm{mL}$ HB-EGF, 10 ng/mL CNTF, 50 ng/mL BMP4, and $20 \mathrm{ng} / \mathrm{mL}$ FGF-2) for 48 hours, followed by resting astrocyte media (1:1 DMEM and Neurobasal Medium with 5ng/ml HB-EGF) for another 72 hours. After this five-day thawing and maturation protocol, experimental treatments could be applied to these mature astrocytes in culture.

\section{Primary screen and secondary dose response screen}

All liquid handling was performed using a BioTek EL406 Washer Dispenser. Astrocytes were thawed and plated, as described for terminal experiments above, onto 384-well plates as described above at a density of 500 cells $/ \mathrm{mm}^{2}$. A Perkin Elmer Janus G3 Varispan Automated Workstation was then used to treat cells with small-molecules with one molecule per well at a concentration of $2 \mathrm{uM}$ in 384-well plates, followed one hour later by $3 \mathrm{ng} / \mathrm{mL}$ IL1 $\alpha$ (Sigma \#I3901), $400 \mathrm{ng} / \mathrm{mL}$ C1q (MyBioSource \#MBS143105), and $30 \mathrm{ng} / \mathrm{mL}$ TNF (R\&D Systems \#210-TA-020). After incubation for 24 hours, the cells were fixed using 4\% paraformaldehyde and stained for GBP2 
(Proteintech \#11854-1-AP) using the procedure detailed in the immunocytochemistry section below, then imaged using the PerkinElmer Operetta CLS High-Content Analysis System. Images were analyzed using automated PerkinElmer Columbus Image Analysis Software. For analysis, toxic chemicals were first removed, a chemical was considered toxic if it decreased the counted number of live cells in the well by greater than $30 \%$ compared to reactive astrocyte plus DMSO vehicle control wells. Hits were then determined as those compounds that decreased the number of GBP2-positive reactive astrocytes by greater than or equal to $90 \%$ compared to reactive astrocyte plus DMSO vehicle control wells.

Secondary dose curve screens were performed exactly as described for the primary screen except with custom generated dose curve plates containing the following drug concentrations: $6 \mathrm{uM}, 3 \mathrm{uM}$, 1.5uM, 0.75uM, 0.37uM, 0.18uM, 0.09uM.

Z-Score Calculation: Z-score is a standard measure of drug screen quality. To calculate the Z' standard where $\sigma=$ standard deviation and $\mu=$ mean of either the positive or negative control wells:

$$
Z^{\prime} \text { standard }=1-\frac{3 \sigma_{\text {positive }}+3 \sigma_{\text {negative }}}{\left|\mu_{\text {positive }}-\mu_{\text {negative }}\right|}
$$

To calculate $Z$ ' Robust where $(\mathrm{mad})=$ mean absolute deviation and $\tilde{x}=$ median of either the positive or negative control wells:

$$
Z^{\prime} \text { robust }=1-\frac{3(\mathrm{mad})_{\text {positive }}+3(\mathrm{mad})_{\text {negative }}}{\left|\tilde{x}_{\text {positive }}-\tilde{x}_{\text {negative }}\right|}
$$

\section{Generation of Hdac3 knockout astrocytes}

To generate $H d a c 3$ knockout astrocytes cells were isolated from $A l d h 1 l 1-C r e E R T^{2} ; H d a c 3^{f l f l}$ mice, generated by crossing Aldh1l1-CreERT 22 (Jax \#031008) and Hdac3 $3^{f l f l}$ (Jax \#024119) $)^{23}$ mice. Aldh1l1-CreERT $T^{2}$ Hdac $3^{f l f l}$ astrocytes were thawed and matured as described above then treated with 1uM tamoxifen (Selleck, S7827) for three days followed by 4-5 days to allow recombination. Cells were then treated with $3 \mathrm{ng} / \mathrm{mL}$ IL1 $\alpha$ (Sigma \#I3901), $400 \mathrm{ng} / \mathrm{mL}$ C1q (MyBioSource \#MBS143105), and $30 \mathrm{ng} / \mathrm{mL}$ TNF (R\&D Systems \#210-TA-020) and 24hrs later fixed and stained as described in immunocytochemistry section below. 


\section{Human astrocyte generation}

Human induced pluripotent stem cells (iPSCs) were cultured to differentiate into astrocytes as previously described ${ }^{34}$. In short, iPSC colonies were placed in neural induction media for 10 days until neural rosettes could be picked, dissociated, and plated in a glial expansion medium. These cells were allowed to proliferate and become a homogenous population of glial progenitor cells (GPCs) over eight passages on poly-L-ornithine and laminin-coated plates. These GPCs were then passaged onto a Matrigel-coated plate to culture in an astrocyte induction media for two weeks, which was followed by culturing for another four weeks in an astrocyte maturation medium.

\section{Real-time polymerase chain reaction (qPCR)}

Cells were lysed in TRIzol and total RNA was extracted with phenol-chloroform followed by spin columns from the RNEasy Mini Kit (Qiagen, 74104). RNA quality and quantity was determined using a NanoDrop spectrophotometer. The RNA was then reverse transcribed using the iScript cDNA synthesis kit (BioRad, 1708891) according to the manufacturer's instructions. Real-time qPCR was performed using the Taqman Gene Expression Master Mix (Applied Biosystems, 4369016) and the Taqman assay probes for human: GBP2 (Thermo Fisher Assay ID: Hs00894837_m1) and PSMB8 (Hs00544758_m1).

\section{ViewRNA ISH in vitro}

In situ hybridization of cells in culture was performed with ThermoFisher ViewRNA according to manufacturer's instructions without modification (ThermoFisher \#QVC0001). Briefly, cells were fixed and permeabilized followed by hybridization with target-specific probes for $2 \mathrm{hrs}$ at $40 \mathrm{C}$. Target probe signal was then amplified and signal was imaged using the PerkinElmer Operetta CLS High-Content Analysis System and analyzed using automated scripts in PerkinElmer Columbus Image Analysis software. Probes for C3 (ThermoFisher, VB4-3112231-VC) and Serping1 (ThermoFisher, VB4-3114232-VC).

\section{Enzyme-linked immunosorbent assay (ELISA) and cytokine profiling}

Secreted cytokine levels were measured by ELISA and cytokine profiling arrays. ELISA assays were performed according to the manufacturer's instructions for CCL5 (R\&D Systems, DY47805) and IL6 (DY406-05). Relative CCL5 and IL6 concentrations were measured based on 
absorbance measured by Synergy Neo2 (BioTek) plate reader. Secreted cytokines were measured using the Proteome Profiler Array Mouse XL Cytokine Array Kit (R\&D Systems, ARY028) according to manufacturer's instructions.

\section{OVA $_{257-264}$ peptide antigen presentation assay}

Mouse primary astrocytes were cultured and treated with reactive astrocyte driving cytokines with or without the HDAC3 specific inhibitors, RGFP966 and T247. After 24 hours of treatment, these cells were cultured in the presence of the OVA257-264 peptide (GenScript RP10611 or Sigma S7951). After 12 hours, the cells were live-stained for two hours using a conjugated antibody targeted against an OVA257-264 peptide antigen (BioLegend, 141605). The cells were then fixed with 4\% paraformaldehyde and imaged using the PerkinElmer Operetta CLS High-Content Analysis System. Images were analyzed using the automated Columbus Image Data Storage and Analysis System to identify the extent to which HDAC3 inhibitors decreased OVA257-264 antigen presentation.

\section{NF-кB Jurkat reporter assay}

Jurkat NF-kB reporter cells were purchased from BPS Bioscience (60651). Cells were grown in manufacturer recommended growth media of RPMI 1640 with 10\% FBS, 1x Pen/Strep, and $1 \mathrm{mg} / \mathrm{mL}$ Geneticin. To test whether drugs inhibit NF-KB activity, the reporter cells were exposed to drugs or DMSO vehicle for one hour before the addition of $3 \mathrm{ng} / \mathrm{mL}$ IL-1a (Sigma, I3901), 400 ng/mL C1q (MyBioSource, MBS143105), and 30 ng/mL TNF (R\&D Systems, 210-TA-020). Cells were then incubated for $24 \mathrm{hrs}$, then luciferase activity was measured with the One-Step Luciferase Assay System (BPS Bioscience, 60690-1) according to manufacturer's instructions with a Synergy Neo2 plate reader (BioTek).

\section{Immunocytochemistry}

For immunocytochemistry, cells were fixed with ice-cold 4\% PFA for 15 minutes at room temperature, washed three times with PBS, blocked and permeabilized with $10 \%$ donkey serum and $0.1 \%$ Triton $\mathrm{X}-100$ in PBS for $1 \mathrm{hr}$, and then stained with primary antibodies overnight followed by three washes with PBS, and then $1 \mathrm{hr}$ incubation with Alexa fluor secondary antibodies and DAPI. Stained cells were imaged using the PerkinElmer Operetta CLS High-Content Analysis 
System and analyzed using automated scripts in PerkinElmer Columbus Image Analysis software. Primary antibodies used were GBP2 (ProteinTech, 11854-1-AP), Vimentin (BioLegend, 919101), AQP4 (Sigma, HPA014784), GLT-1 (Novus Biologicals, NBP1-20136), ALDH1L1 (Novus Biologicals, NBP2-50045), and HDAC3 (BioLegend, 685202).

\section{Single-cell RNAseq sample preparation and analysis}

Resting and reactive astrocytes were lifted from culture plates using TrypLE (ThermoFisher, 12563011) and collected in 1x PBS with 1\% bovine serum albumin (BSA). Cells were then spun down at $1000 \mathrm{rpm}$ for 10 minutes. After which cells were washed once with 1x PBS with 1\% BSA before being filtered through 40um FlowMi Tip Strainers (VWR, 10032-802). Cells were then diluted with 1x PBS with 1\% BSA and loaded onto the Chromium 10x Controller according to manufacturer's instructions. Following partitioning of single-cells in gel bead emulsions, reversetranscriptions and library preparation were performed according to 10x Single Cell 3' v2 chemistry kit instructions (v2 kit since discontinued). Finally, libraries were sequenced by the Case Western Reserve University Genomics Core on an Illumina HiSeq2500 with paired-end 50bp reads and a target sequence depth of 50,000 reads per cell. Sequence data were first processed by 10x Cell Ranger v3.1 using default settings to generate a gene expression matrix and then all downstream analysis was performed with the R package, Seurat v4.0 ${ }^{12}$.

\section{Bulk RNAseq sample preparation and analysis}

Total RNA was extracted from resting and reactive astrocytes using the same procedure as described for qPCR and sent to Novogene for mRNA Sequencing. For gene expression analysis, reads were mapped to the mm10 genome using kallisto v0.46.1 (https://pachterlab.github.io/kallisto/) ${ }^{13}$. Transcripts were summarized to the gene level with tximport v1.2 (https://bioconductor.org/packages/release/bioc/html/tximport.html) ${ }^{14}$. Normalized expression and differential gene expression were then generated using DESeq2 v1.32.0 (https://bioconductor.org/packages/release/bioc/html/DESeq2.html) ${ }^{15}$.

\section{H3K27ac and RelA/P65 Chromatin immunoprecipitation sequencing (ChIP-seq)}

Nuclei isolation and chromatin shearing were performed using the Covaris TruChIP protocol following manufacturer's instructions for the "high-cell" format. In brief, 5 million (H3K27Ac) or 
20 million resting and reactive (RelA/P65) were cross-linked in "Fixing buffer A" supplemented with $1 \%$ fresh formaldehyde for 10 minutes at room temperature with oscillation then quenched for 5 minutes with "Quench buffer E." These cells were then washed with PBS and either snap frozen and stored at $-80 \mathrm{C}$ or immediately used for nuclei extraction and shearing per the manufacturer protocol. The samples were sonicated with the Covaris S2 using the following settings: 5\% Duty factor 4 intensity for four 60 seconds cycles. Sheared chromatin was cleared and incubated overnight at $4 \mathrm{C}$ with primary antibodies that were pre-incubated with protein $\mathrm{G}$ magnetic DynaBeads (Thermo Fisher, 10004D). Primary antibodies used included anti-H3K27Ac (Abcam, ab4729) and anti-RelA/P65 (Cell Signaling Technology, 8242). These beads were then washed, eluted, reverse cross-linked, and treated with Rnase A followed by proteinase K. ChIP DNA was purified using Ampure XP beads (Aline Biosciences, C-1003-5) and then used to prepare Illumina sequencing libraries as described previously ${ }^{16}$. Libraries were sequenced on the Illumina HiSeq2500 with single-end 50bp reads with a read-depth of at least 20 million reads per sample.

For peak calling, reads were quality and adaptor trimmed using Trim Galore! Version 0.4.1. Trimmed reads were aligned to mm10 with Bowtie2 version 2.3.217. Duplicate reads (potential artifacts of PCR in library preparation) were removed using Picard MarkDuplicates. Peaks were called with MACS version 2.1.1 ${ }^{18}$. Peaks were visualized with the Integrative Genomics Viewer (IGV, Broad Institute). Peaks were compared and contrasted using bedtools implemented in the R software environment using the BedtoolsR package (http://phanstiellab.med.unc.edu/bedtoolsr.html) and peaks were assigned to the nearest gene using the R packages ChIPSeeker $^{19}$, ChIPpeakanno ${ }^{20}$, and GenomicRanges ${ }^{21}$. Super-enhancers were called using rank ordering of super-enhancers (ROSE) analysis package ${ }^{24,25}$. Super-enhancers and genes targeted by super-enhancers were generated for the H3K27ac biological replicate with the strongest signal and key findings confirmed with a second biological replicate.

\section{Omni Assay for Transposase-Accessible Chromatin using sequencing (ATAC-seq)}

Omni ATAC-Seq was performed on 50,000 resting and reactive astrocytes following the protocol outlined in Corces et al. ${ }^{22}$ In brief, nuclei were extracted from cells and treated with transposition mixture containing Nextera Tn5 Transposase for (Illumina, FC-121-1030). Transposed fragments 
were then purified using QIAGEN MinElute columns (QIAGEN, 28004), PCR amplified, and libraries were purified with PCRClean DX purification system (Aline Biosciences, C-1003-5) with a sample to bead ratio of 1:1.2. Final libraries were sequenced on the illumina HiSeq2500 with single-end 50bp reads and nearly 100 million reads per sample. Reads were aligned to the mm 10 mouse genome following the same pipeline used for ChIP-seq data (see ChIP-seq and analysis) and peaks were called using the "narrowPeaks" function of MACS version 2.1.1, as outlined for RelA/P65 ChIP-seq (see ChIP-seq and analysis).

\section{Motif enrichment analysis}

Motifs were called under RelA/P65 peaks, ATAC-seq peaks, or regions of gained or lost H3K27Ac using HOMERv4.11.1 (Heinz et al., 2010). The findMotifsGenome.pl tool was used with mm10 as the reference genome.

\section{Pharmacokinetics}

C57BL/6 adult mice were injected i.p. with $10 \mathrm{mg} / \mathrm{kg}$ RGFP966 daily for 11 days. Mice were then perfused with saline to remove blood from the brain. Brain tissue was then collected and snap frozen. Brain tissues were thawed at room temperature and homogenized in PBS. Calibration standards and study samples were extracted with $3 \mathrm{x}$ volume of acetonitrile containing $0.1 \%$ formic acid with $200 \mathrm{ng} / \mathrm{ml}$ internal standard solution. Samples were then each vortexed for 1 minute, then transferred to an Eppendorf R5417R and centrifuged at $14000 \mathrm{rpm}$ for 7 minutes. Following extraction of tissue, homogenate calibrators and study samples were transferred directly to an autosampler microtiter plate for analysis. Samples were analyzed by LC-MS-MS in the positive ion electrospray ionization mode.

\section{Immunohistochemistry}

Mice were perfused with PBS followed by $4 \%$ paraformaldehyde, after which brains were extracted and cryopreserved in 30\% sucrose, then frozen in OCT and sectioned. To stain, slides were washed with PBS and then incubated overnight with primary antibody. After primary incubation, slides were then washed and labeled with Alexa Fluor secondary antibodies (ThermoFisher). Images were captured on a Hamamatsu Nanozoomer S60 Digital slide scanner with NDPview 2.0 software. Image analysis was performed using automated scripts with Perkin 
Elmer Columbus Image Analysis Software. Primary antibodies used were AcH4 (EMD Millipore, 06-866), GFAP (DAKO, 685202), and IBA-1 (Abcam, 685202)

\section{Western Blotting}

Protein samples were collected in RIPA buffer (Sigma, R0278) with Halt Protease and Phosphatase Inhibitor (ThermoFisher, 78441). Total protein concentration was determined by BCA assay (ThermoFisher, 23225). Equal amounts of total protein were loaded onto 4-12\% BisTris gels (Invitrogen). Proteins were then separated by gel-electrophoresis and transferred to PVDF membranes (ThermoFisher, LC2002). Blots were probed with RelA/p65 (Cell Signaling Technology, 8242) and beta-Actin (Sigma, A3854), developed using SuperSignal West Pico Plus Chemiluminescent Substrate (ThermoFisher, 34577), and visualized using a Li-Cor Odyssey XF Imager.

\section{In situ hybridization with RNAscope}

In situ hybridization for in vivo studies was performed using RNAscope Multiplex Fluorescence V2 Assay (ACD Bio, 323136) according to manufacturer's instructions for fixed frozen samples. Briefly, tissue was prepared by first dehydrating with increasingly higher percentages of ethanol, then dried, blocked with hydrogen peroxide, followed by antigen retrieval for 5 minutes, dried again, and then protein was digested using provided Protease III. RNA-Targeting probes purchased from ACD Bio were then annealed at 40C for 2 hours followed by washing and a series of amplification steps before finally tagging the RNA with Opal Dye fluorophores (Perkin Elmer). In situ hybridization Images were captured on a Hamamatsu Nanozoomer S60 Digital Slide scanner with NDPview 2.0 software. Image analysis was performed using automate scrips with Perkin Elmer Columbus Image Analysis Software. The following mouse specific RNAscope probes were used: Slcla3 (ACD Bio, 430781), Gbp2 (ACD Bio, 572491), Gfap (ACD Bio, 313211), and C3 (ACD Bio, 417841).

\section{Electron microscopy sample preparation and analysis}

Samples were processed as previously described ${ }^{23}$. Briefly, mice were perfused with $4 \%$ PFA, $2 \%$ gluteraldehyde, and $0.1 \mathrm{M}$ sodium cacodylate. Spinal cords were extracted, and samples were osmicated, stained en bloc with uranyl acetate and embedded in Embed 812, an Epon-812 
substitute (Electron Microscopy Sciences). Thin sections were cut, carbon-coated and imaged either on a Helios NanoLab 660 Scanning Electron Microscope (FEI)

\section{Gene ontology (GO) and gene set enrichment analysis (GSEA)}

GO analysis was performed using gProfiler (https://biit.cs.ut.ee/gprofiler/gost) ${ }^{24}$ with a significance threshold set at false discovery rate $($ FDR $)<0.05$ and calculated by BenjaminiHochberg FDR. Redundant GO terms were removed using REVIGO ${ }^{35}$ (http://revigo.irb.hr/) using default settings. Comparisons between RelA/p65 direct target genes and non-RelA/p65 direct target genes GO terms was performed with the $\mathrm{R}$ package, clusterProfiler ${ }^{36}$ v4.0.5 (https://bioconductor.org/packages/release/bioc/html/clusterProfiler.html), following the tutorial for "Biological Theme Comparison" at https://yulab-smu.top/biomedical-knowledge-miningbook/index.html. GSEA scores were generated for curated gene sets from publicly-available data. GSEA was run using classical scoring, 1000 gene-set permutations, and signal-to-noise metrics. Normalized enrichment scores (NES) and FDR were calculated by the GSEA software ${ }^{37}$ (https://www.gsea-msigdb.org/gsea/index.jsp).

\section{Statistical analysis}

Unless otherwise noted, GraphPad Prism was used to perform statistical analyses The statistical tests used and description of data presentation and sample size can be found in each figure legend. Unless otherwise noted, sample sizes were determined by reference to previous literature. For all Tukey box-and-whisker plots, the middle line of the box is the median, the box extends from the $25^{\text {th }}$ to $75^{\text {th }}$ percentile, the upper whisker is placed at the $75^{\text {th }}$ percentile plus $1.5 \mathrm{x}$ the inter-quartile distance, the lower whisker is placed at the $25^{\text {th }}$ percentile plus $1.5 \mathrm{x}$ the inter-quartile distance, and any individual data points that fall outside of the upper and lower whiskers are plotted.

\section{Data availability}

All datasets generated in this study have been deposited in Gene Expression Omnibus (https://www.ncbi.nlm.nih.gov/geo/) under SuperSeries accession code GSE185215 with subseries for RNA-seq (GSE185212), scRNA-seq (GSE184437), ChIP-seq (GSE185606), and ATAC-seq (GSE185605). 
Acknowledgments This study was supported by grants from the National Institutes of Health R35NS116842 (P.J.T.), F30HD096784 (K.C.A.), and T32GM007250 (K.C.A), and the Hartwell Foundation (B.L.L.C), as well as institutional support from the Case Western Reserve University School of Medicine and philanthropic support from the Fakhouri, R. Blane \& Claudia Walter, Long, Goodman, Geller, and Weidenthal families, and the Research Institute for Children's Health.

Additional support was provided by the Small Molecule Drug Development and Genomics core facilities of the CWRU Comprehensive Cancer Center (P30CA043703), the CWRU Light Microscopy Imaging Center (S10-OD016164), and the University of Chicago Genomics Facility. The authors thank Drew Adams, Yuriy Federov, Bill Harte, Marissa Scavuzzo, Erin Cohn, Mayur Madhavan, and Matt Elitt for technical assistance and/or discussion.

Author contributions B.L.L.C. and P.J.T. conceived and managed the overall study. B.L.L.C. and J.D.K. performed, quantified, and analyzed all in vitro studies. H.E.S. helped to generate astrocyte cultures. B.L.L.C. performed and analyzed all single-cell RNAseq experiments. B.L.L.C. performed the small-molecule screen and all validations. K.C.A. performed all ChIP-seq and ATAC-seq experiments and super-enhancer analysis. B.L.L.C. performed ChIP-seq, ATAC-seq, and bulk RNA-seq data analysis. B.L.L.C. and A.M.S performed and analyzed in vivo LPS studies. M.K., E.G., and R.H.M. performed in vivo LPC studies and generated electron microscopy images. Y.M.H. analyzed electron microscopy images. B.L.L.C. assembled all figures. B.L.L.C. and P.J.T. wrote the manuscript with input from all authors.

Competing interests B.L.L.C. and P.J.T. are listed as inventors on pending patent claims filed by Case Western Reserve University covering methods and compositions for treating neurodegenerative disorders. All other authors declare no competing interests. 


\section{References}

1 Liddelow, S. A. et al. Neurotoxic reactive astrocytes are induced by activated microglia. Nature 541, 481-487, doi:10.1038/nature21029 (2017).

2 Clarke, L. E. et al. Normal aging induces A1-like astrocyte reactivity. Proc Natl Acad Sci U S A 115, E1896-E1905, doi:10.1073/pnas.1800165115 (2018).

3 Wheeler, M. A. et al. MAFG-driven astrocytes promote CNS inflammation. Nature 578, 593-599, doi:10.1038/s41586-020-1999-0 (2020).

4 Smith, H. L. et al. Astrocyte Unfolded Protein Response Induces a Specific Reactivity State that Causes Non-Cell-Autonomous Neuronal Degeneration. Neuron 105, 855-866 e855, doi:10.1016/j.neuron.2019.12.014 (2020).

5 Yun, S. P. et al. Block of A1 astrocyte conversion by microglia is neuroprotective in models of Parkinson's disease. Nat Med 24, 931-938, doi:10.1038/s41591-018-0051-5 (2018).

6 Guttenplan, K. A. et al. Neurotoxic reactive astrocytes induce cell death via saturated lipids. Nature, doi:10.1038/s41586-021-03960-y (2021).

7 Hasel, P. \& Liddelow, S. A. Astrocytes. Curr Biol 31, R326-R327, doi:10.1016/j.cub.2021.01.056 (2021).

8 Allen, N. J. \& Eroglu, C. Cell Biology of Astrocyte-Synapse Interactions. Neuron 96, 697708, doi:10.1016/j.neuron.2017.09.056 (2017).

9 Nagai, J. et al. Behaviorally consequential astrocytic regulation of neural circuits. Neuron 109, 576-596, doi:10.1016/j.neuron.2020.12.008 (2021).

10 Dooves, S. et al. Astrocytes are central in the pathomechanisms of vanishing white matter. J Clin Invest 126, 1512-1524, doi:10.1172/JCI83908 (2016).

11 Absinta, M. et al. A lymphocyte-microglia-astrocyte axis in chronic active multiple sclerosis. Nature 597, 709-714, doi:10.1038/s41586-021-03892-7 (2021).

12 Leng, K. et al. CRISPRi screens in human astrocytes elucidate regulators of distinct inflammatory reactive states. BioRxiv, 2021.2008.2023.457400, doi:10.1101/2021.08.23.457400 (2021).

13 Najm, F. J. et al. Drug-based modulation of endogenous stem cells promotes functional remyelination in vivo. Nature 522, 216-220, doi:10.1038/nature14335 (2015).

14 Foo, L. C. et al. Development of a method for the purification and culture of rodent astrocytes. Neuron 71, 799-811, doi:10.1016/j.neuron.2011.07.022 (2011).

15 Grubman, A. et al. A single-cell atlas of entorhinal cortex from individuals with Alzheimer's disease reveals cell-type-specific gene expression regulation. Nat Neurosci 22, 2087-2097, doi:10.1038/s41593-019-0539-4 (2019).

16 Al-Dalahmah, O. et al. Single-nucleus RNA-seq identifies Huntington disease astrocyte states. Acta Neuropathol Commun 8, 19, doi:10.1186/s40478-020-0880-6 (2020). 
17 Smajić, S. et al. Single-cell sequencing of the human midbrain reveals glial activation and a neuronal state specific to Parkinson's disease. medRxiv, 2020.2009.2028.20202812, doi:10.1101/2020.09.28.20202812 (2020).

18 Chastain, E. M., Duncan, D. S., Rodgers, J. M. \& Miller, S. D. The role of antigen presenting cells in multiple sclerosis. Biochim Biophys Acta 1812, 265-274, doi:10.1016/j.bbadis.2010.07.008 (2011).

19 Hartmann, K. et al. Complement 3(+)-astrocytes are highly abundant in prion diseases, but their abolishment led to an accelerated disease course and early dysregulation of microglia. Acta Neuropathol Commun 7, 83, doi:10.1186/s40478-019-0735-1 (2019).

20 Malvaez, M. et al. HDAC3-selective inhibitor enhances extinction of cocaine-seeking behavior in a persistent manner. Proceedings of the National Academy of Sciences 110, 2647-2652, doi:10.1073/pnas.1213364110 (2013).

21 Suzuki, T. et al. Identification of highly selective and potent histone deacetylase 3 inhibitors using click chemistry-based combinatorial fragment assembly. PLOS ONE 8, e68669, doi:10.1371/journal.pone.0068669 (2013).

22 Srinivasan, R. et al. New Transgenic Mouse Lines for Selectively Targeting Astrocytes and Studying Calcium Signals in Astrocyte Processes In Situ and In Vivo. Neuron 92, 1181-1195, doi:10.1016/j.neuron.2016.11.030 (2016).

23 McQuown, S. C. et al. HDAC3 is a critical negative regulator of long-term memory formation. J Neurosci 31, 764-774, doi:10.1523/JNEUROSCI.5052-10.2011 (2011).

24 Loven, J. et al. Selective inhibition of tumor oncogenes by disruption of super-enhancers. Cell 153, 320-334, doi:10.1016/j.cell.2013.03.036 (2013).

25 Whyte, W. A. et al. Master transcription factors and mediator establish super-enhancers at key cell identity genes. Cell 153, 307-319, doi:10.1016/j.cell.2013.03.035 (2013).

26 Hnisz, D. et al. Super-enhancers in the control of cell identity and disease. Cell 155, 934947, doi:10.1016/j.cell.2013.09.053 (2013).

27 Molofsky, A. V. \& Deneen, B. Astrocyte development: A Guide for the Perplexed. Glia 63, 1320-1329, doi:10.1002/glia.22836 (2015).

28 Vargas, M. R., Johnson, D. A., Sirkis, D. W., Messing, A. \& Johnson, J. A. Nrf2 activation in astrocytes protects against neurodegeneration in mouse models of familial amyotrophic lateral sclerosis. J Neurosci 28, 13574-13581, doi:10.1523/JNEUROSCI.4099-08.2008 (2008).

29 Tonelli, C., Chio, I. I. C. \& Tuveson, D. A. Transcriptional Regulation by Nrf2. Antioxid Redox Signal 29, 1727-1745, doi:10.1089/ars.2017.7342 (2018).

30 Hasel, P., Rose, I. V. L., Sadick, J. S., Kim, R. D. \& Liddelow, S. A. Neuroinflammatory astrocyte subtypes in the mouse brain. Nat Neurosci, doi:10.1038/s41593-021-00905-6 (2021).

31 Zamanian, J. L. et al. Genomic analysis of reactive astrogliosis. J Neurosci 32, 6391-6410, doi:10.1523/JNEUROSCI.6221-11.2012 (2012). 
32 El Waly, B., Buttigieg, E., Karakus, C., Brustlein, S. \& Debarbieux, F. Longitudinal Intravital Microscopy Reveals Axon Degeneration Concomitant With Inflammatory Cell Infiltration in an LPC Model of Demyelination. Front Cell Neurosci 14, 165, doi:10.3389/fncel.2020.00165 (2020).

33 McMurran, C. E., Zhao, C. \& Franklin, R. J. M. Toxin-Based Models to Investigate Demyelination and Remyelination. Methods Mol Biol 1936, 377-396, doi:10.1007/978-14939-9072-6_21 (2019).

34 Perriot, S. et al. Human Induced Pluripotent Stem Cell-Derived Astrocytes Are Differentially Activated by Multiple Sclerosis-Associated Cytokines. Stem Cell Reports 11, 1199-1210, doi:10.1016/j.stemcr.2018.09.015 (2018).

$36 \mathrm{Wu}, \mathrm{T}$. et al. clusterProfiler 4.0: A universal enrichment tool for interpreting omics data. Innovation (N Y) 2, 100141, doi:10.1016/j.xinn.2021.100141 (2021).

37 Subramanian, A. et al. Gene set enrichment analysis: a knowledge-based approach for interpreting genome-wide expression profiles. Proc Natl Acad Sci U S A 102, 1554515550, doi:10.1073/pnas.0506580102 (2005). 
662

663

Figure 1
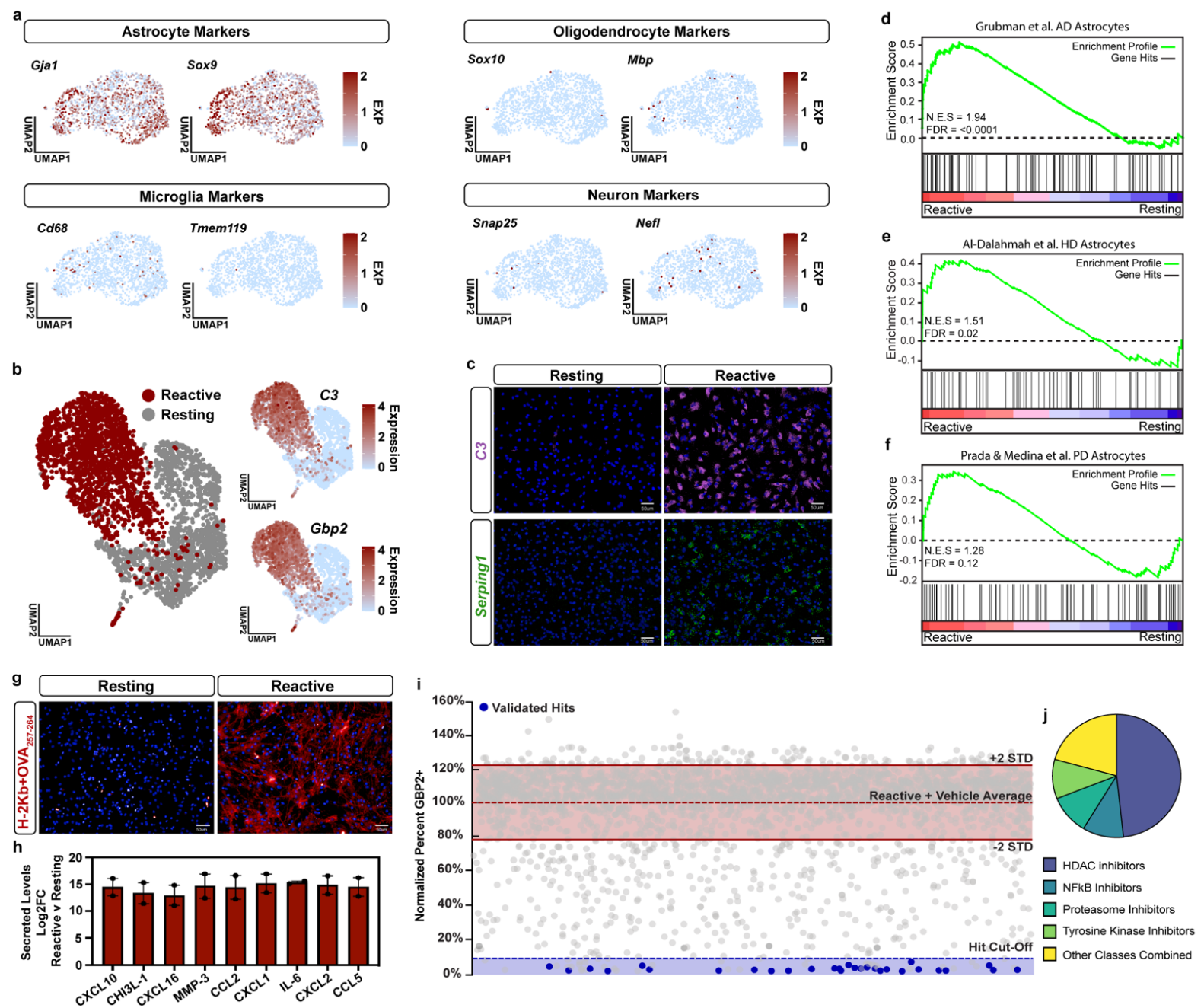

Figure 1. An astrocyte discovery platform identifies inhibitors of pathological reactive astrocyte formation. a, Expression of cell-type specific markers layered onto UMAP plots from single-cell RNAseq of primary resting astrocytes. b, UMAP plot of resting and pathological reactive astrocytes (abbreviated to reactive in figures) single-cell RNAseq colored by condition, resting (grey) and reactive (red). Additional UMAP plots showing expression levels for the pathological reactive astrocyte markers C3 and Gbp2. c, Representative images of in situ hybridization in resting and reactive astrocyte cultures with probes against the pathological reactive astrocyte markers $C 3$ and Serping1. d-f, Gene set enrichment analysis (GSEA) comparing pathological reactive astrocytes to the top 100 genes upregulated in astrocytes from single-nuclei RNAseq data from d, Alzheimer's, e, Huntington's, and f, Parkinson's disease patient tissue. $\mathbf{g}$, Representative images of resting and reactive astrocyte cultures exposed to the $\mathrm{OVA}_{257-264}$ peptide and then stained for MHC Class I bound to $\mathrm{OVA}_{257-264}\left(\mathrm{H}-2 \mathrm{~Kb}+\mathrm{OVA}_{257-264}\right)$ in red. $\mathbf{h}$, The Log2 fold-change (Log2FC) of secreted cytokines in reactive vs resting astrocytes conditioned media. Data presented as mean \pm s.e.m for $n=2$ biological replicates (independent astrocyte isolations). i, Scatter plot of primary screen results displayed as percent GBP2 positive, 
681 normalized to reactive astrocyte plus vehicle controls for all non-toxic chemicals and validated hit 682 chemicals colored in blue. The dashed blue line represents the hit cut-off at a $\geq 90 \%$ decrease in 683 GBP2- positive astrocytes compared to reactive astrocyte plus vehicle controls. Dashed red line 684 represents the average percent GBP2 positive for reactive astrocytes plus vehicle set at $100 \%$. 685 Solid lines represent $+/$ - 2 standard deviations from the mean of reactive plus vehicle control wells. $686 \mathrm{j}$, Pie chart depicting the chemical class breakdown of all 29 validated chemical hits. 
687

688

689

690

691

692

693

694

695

696

697

698

\section{Extended Data Figure 1}
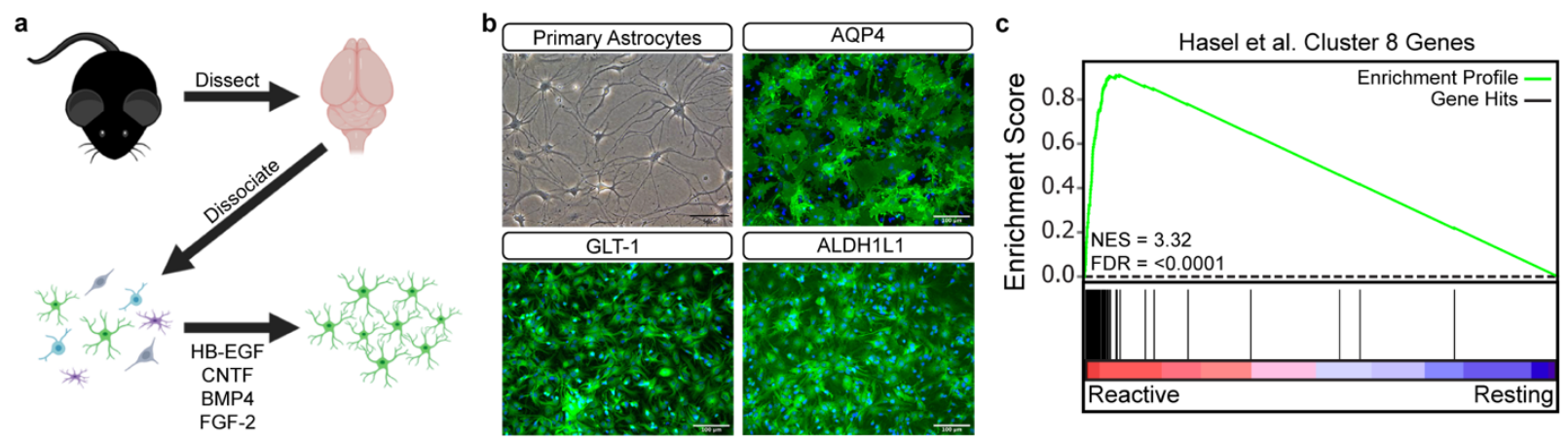

\section{Extended Data Figure 1. Generation of primary mouse astrocytes and correlation with in} vivo reactive astrocyte subtypes. a, Overview diagram of astrocyte isolation and enrichment protocol. b, Phase contrast image showing prototypical astrocyte morphology. Scale bar is $50 u m$. Immunofluorescence images showing expression of canonical astrocyte markers AQP4, GLT-1 (SLC1A2), and ALDH1L1. Scale bar is 100um. c, GSEA comparing pathological reactive astrocytes to Hasel et al. (Nature Neuroscience 2021) Cluster 8 defining genes. Cluster 8 represents a reactive astrocyte state specific to neuroinflammation that also reflects reactive astrocytes in disease models. 


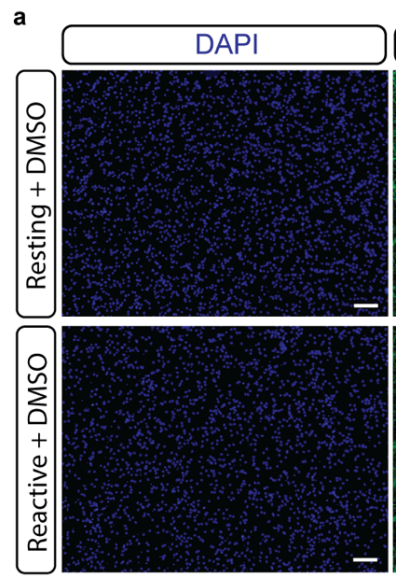

b

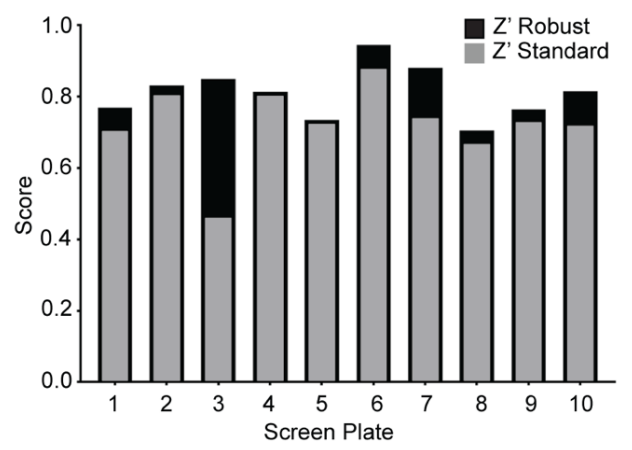

d

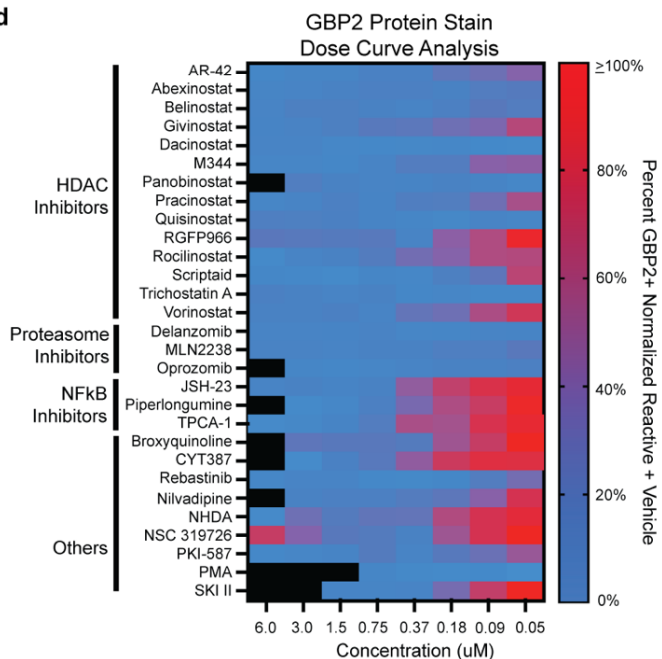

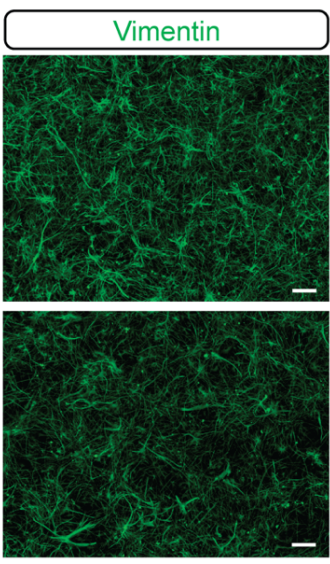
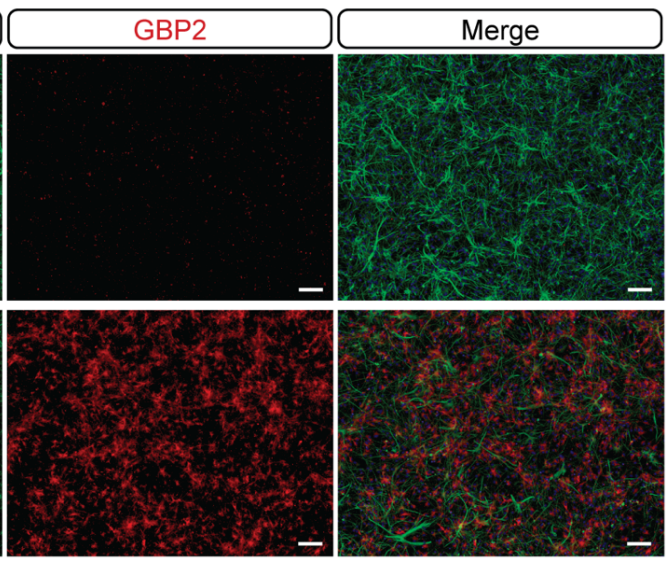

c

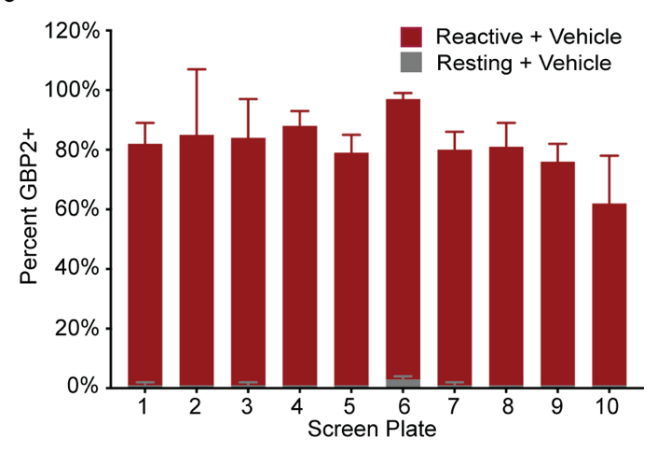

e

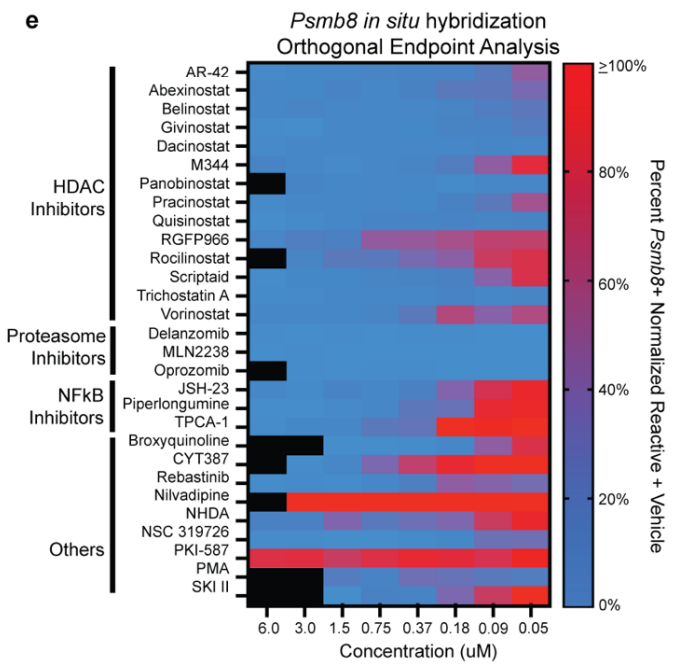

Extended Data Figure 2. Primary screen quality control and secondary validation of primary hits. a, Example images of DMSO vehicle-treated reactive and resting control wells. Scale bar is 100 um. b, Z-prime standard, and robust scores for each primary screen plate. c, Average percent GBP2 positive astrocytes in DMSO vehicle treated reactive and resting control wells on each primary screen plate. d, Dose curve analysis of hits from primary screen. Compounds were tested across an 8-point dose curve with decreasing half-steps from 6uM to 
$7090.05 \mathrm{uM}$. Data are presented as the percent of GBP2 positive cells normalized to DMSO vehicle

710 treated reactive astrocyte control wells. $n=2$ biological replicates (independent astrocyte

711 isolations). Black data points represent toxic doses where total cell number in the well decreased

712 by $>50 \%$ compared to DMSO vehicle treated reactive astrocyte control wells. e, Dose curve

713 analysis of hits from primary screen with Psmb8 positivity by in situ hybridization as a secondary

714 endpoint. Compounds were tested across an 8-point dose curve with decreasing half-steps from

$7156 \mathrm{uM}$ to $0.05 \mathrm{uM}$. Data are presented as the percent of Psmb8 positive normalized to DMSO vehicle

716 treated reactive astrocyte control wells with an $n=1$ biological replicate (independent astrocyte

717 isolation). Black data points represent toxic doses where total cell number in the well decreased

718 by $>50 \%$ compared to DMSO vehicle treated reactive astrocyte control wells. 
a

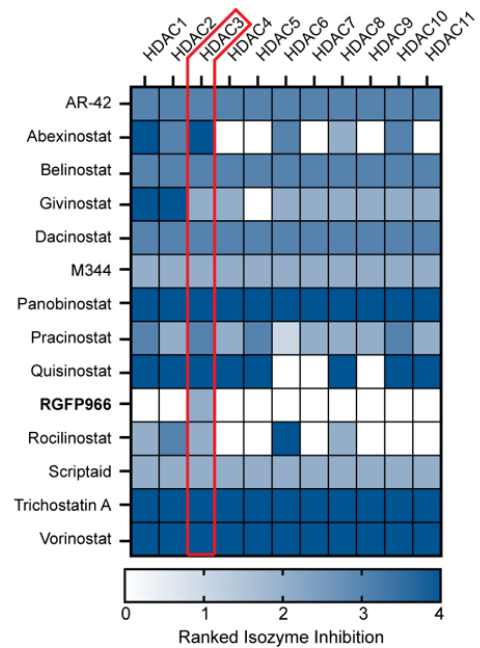

d

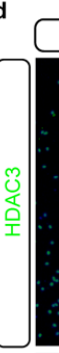

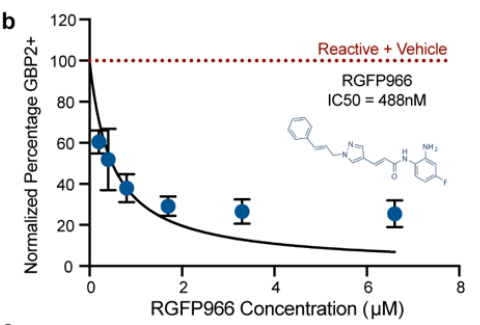

c

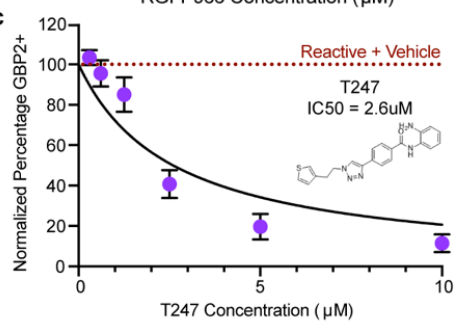

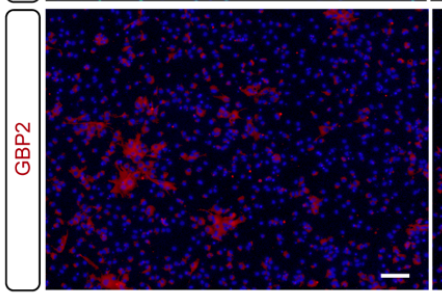
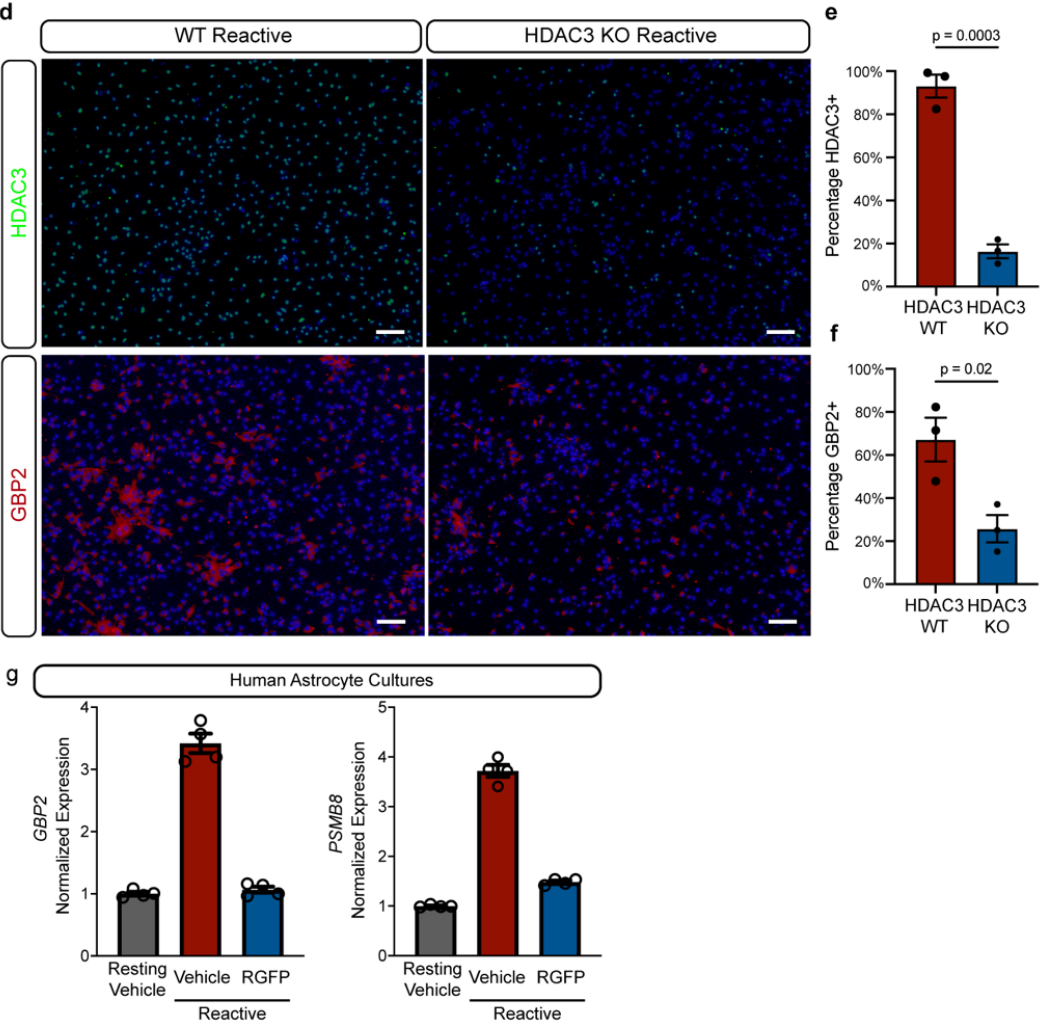

Extended Data Figure 3. Pharmacological and genetic inhibition of HDAC3 modulates pathological reactive mouse and human astrocytes. a, Ranked inhibition against each HDAC isozyme for the validated HDAC inhibitors identified in the primary screen. Highlighted is HDAC3, which is the only shared target between all HDAC inhibitor hits from the primary screen. Ranked efficiency was pulled from target data provided by Selleck Chemical for each compound. b, Dose curve and IC50 value for the HDAC3 specific inhibitor RGFP966. c, Dose curve and IC50 value for the HDAC3 specific inhibitor T247. d, Representative images of wild-type (WT) and HDAC3 
731 is 100um. e, Quantification of the percentage of cells positive for HDAC3 normalized to WT for 732 the experiment represented in d. Data are presented as the mean \pm s.e.m. for $n=3$ biological 733 replicates (independent astrocyte isolations). p-value generated with a Student's two-tailed t-test. 734 f, Quantification of the percentage of cells positive for GBP2 normalized to WT for the experiment 735 represented in $\mathrm{d}$. Data are presented as the mean \pm s.e.m. for $n=3$ biological replicates 736 (independent astrocyte isolations). p-value generated with a Student's two-tailed t-test. g, GBP2 737 and PSMB8 qPCR results for human iPSC derived resting or reactive (TNF, IL1 $\alpha$, and C1q 738 treated) astrocyte cultures treated with vehicle or 5uM RGFP966. Data are presented as mean \pm 739 s.e.m. for $n=4$ technical replicates. 


\section{Figure 2}
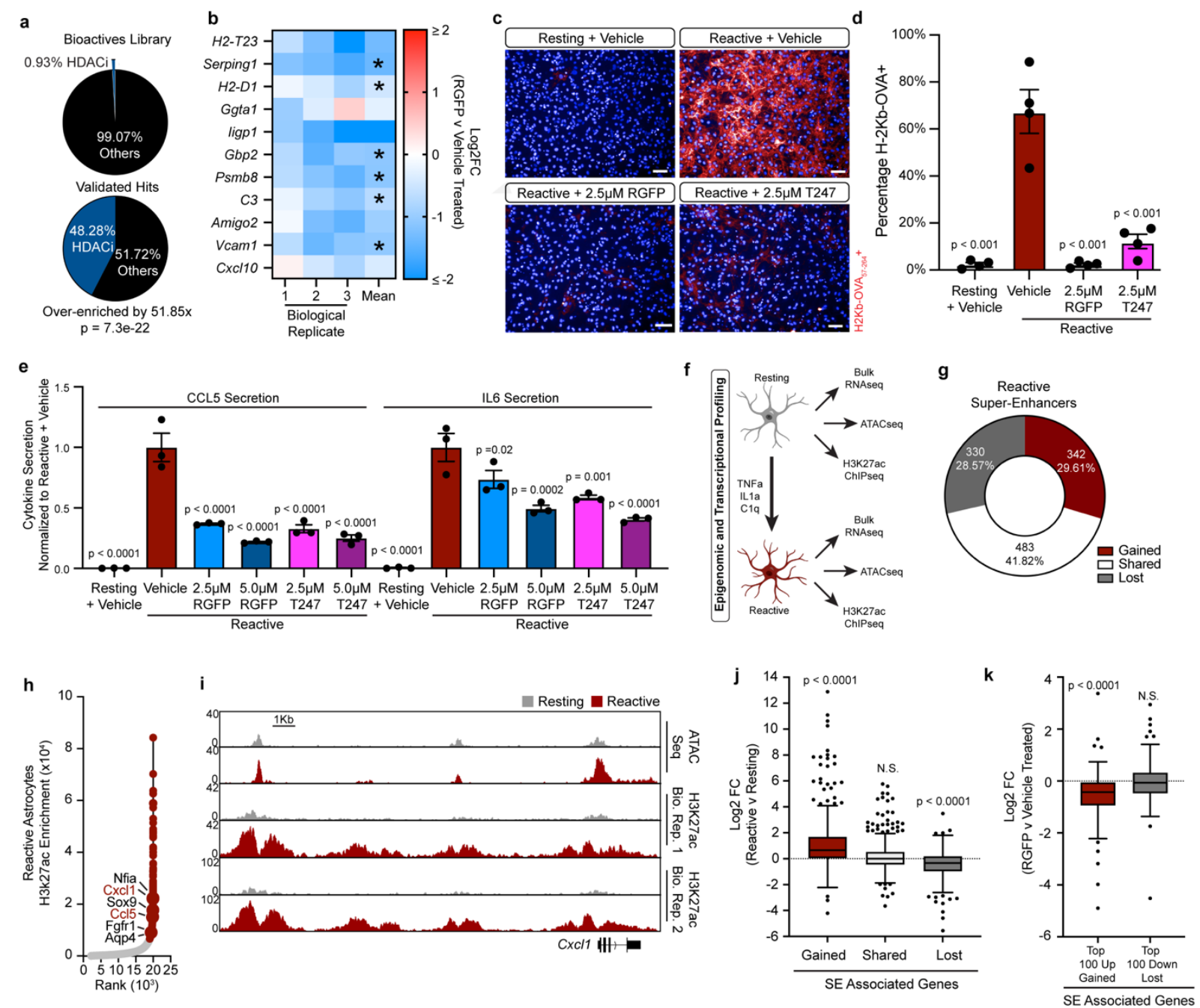

Figure 2. Chemical inhibition of HDAC3 modulates pathological reactive astrocytes. a, Proportion of HDAC inhibitor compounds enriched in primary screen validated hit list compared to the primary screen chemical library as a whole, showing that HDAC inhibitors are significantly enriched in the validated hit list. $p$-value generated by a hypergeometric test. $\mathbf{b}$, Heatmap of the Log2 fold-change (Log2FC) between RGFP966 (RGFP) and vehicle (DMSO) treated pathological reactive astrocytes. Red is upregulated and blue is downregulated in RGFP treated cells. Data are presented as Log2FC for $n=3$ biological replicates (independent astrocyte isolations) with mean and asterisks denoting a $p<0.05$ calculated by DESeq2. c, Representative images of resting and reactive astrocytes treated with vehicle or the HDAC3 inhibitors RGFP or T247, then exposed to the OVA $257-264$ peptide and stained for MHC Class I bound to OVA $257-264(\mathrm{H}-2 \mathrm{~Kb}+\mathrm{OVA})$ in red. Scale bar is $50 \mu \mathrm{m}$. d, Quantification of experiments represented in c. Data presented as mean \pm s.e.m. $n=4$ biological replicates (independent astrocyte isolations). p-values generated by a one-way ANOVA with Dunnett post-test for multiple comparisons to reactive plus vehicle control. e) Quantification of CCL5 and IL6 ELISAs performed on astrocyte conditioned media. 
Data presented as mean \pm s.e.m for an $n=3$ biological replicates (independent astrocyte isolations). p-value generated by a one-way ANOVA with Dunnett post-test for multiple comparisons to reactive plus vehicle control. f, Diagram of transcriptional and epigenetic data captured to analyze chromatin changes between resting and pathological reactive astrocytes. $\mathbf{g}$, Distribution of gained, shared, and lost super-enhancers in pathological reactive astrocytes. $\mathbf{h}$, Hockey-stick plot showing H3K27ac enrichment at enhancers in pathological reactive astrocytes. The enhancers are ranked and those in red were called as super-enhancers by ROSE analysis. Closest genes to each super-enhancer were called with HOMER, genes in red are targeted by gained super-enhancers in pathological reactive astrocytes while those in black are targeted by shared super-enhancers that were called in both resting and pathological reactive astrocytes. i, Example browser track for the gained super-enhancer gene Cxc/1. j, Tukey box and whisker plot showing the average Log2FC between pathological reactive and resting astrocytes for genes associated with gained, shared, and lost super-enhancers in pathological reactive astrocytes. pvalue is generated with a one-sample Wilcoxon Signed Ranked test comparing to a hypothetical median of $\log 2 \mathrm{FC}=0$, which would designate no difference in expression between reactive and resting astrocytes. $\mathbf{k}$, Tukey box and whisker plot showing the average Log2FC between RGFP and vehicle treated pathological reactive astrocytes for the top 100 upregulated genes associated with gained super-enhancers and the top 100 downregulated genes associated with lost superenhancers in pathological reactive astrocytes. $p$-value is generated with a one-sample Wilcoxon Signed Ranked test comparing to a hypothetical median of Log2FC $=0$ which would designate no difference in expression between reactive and resting astrocytes. 
a

\begin{tabular}{|c|c|c|}
\hline \multicolumn{3}{|c|}{ Reactive Super-Enhancers } \\
\hline TF & MOTIF & p-value \\
\hline RelA/p65 & GGAAATICCC & $1 e-6$ \\
\hline Atf3 & 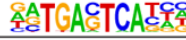 & $1 e-6$ \\
\hline RelA/p65 & AGGGGATTTCC: & $1 e-5$ \\
\hline Atf7 & 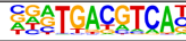 & $1 e-4$ \\
\hline
\end{tabular}

b

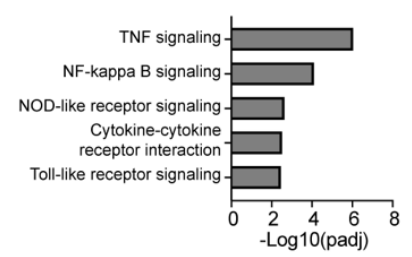

f

beta-Actin $(42 \mathrm{kDa})$ Sigma Cat\# A3854

Lanes:

1. Resting

2. Reactive

3. Reactive + RGFP966

4. Reactive $+\mathrm{T} 247$
5. Resting

6. Reactive

7. Reactive + RGFP966

8. Reactive + T247
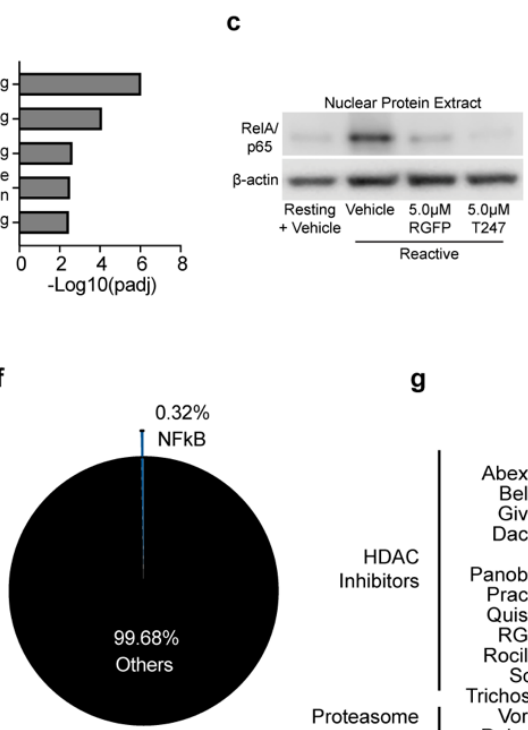

d

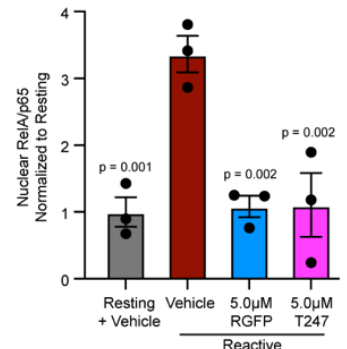

g

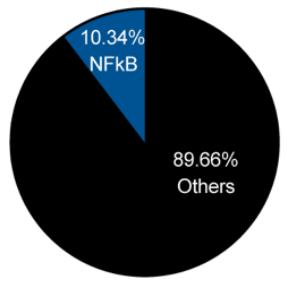

Over-enrichment $=32.22 x$ $p=8.34 \mathrm{e}-5$

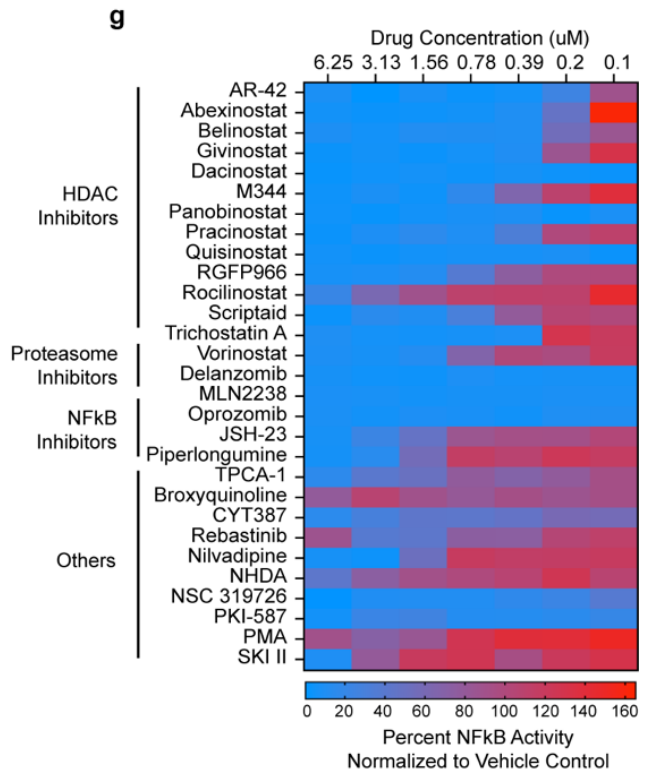

Extended Data Figure 4. RelA/p65 regulates pathological reactive astrocyte formation and is a unifying target of small-molecule inhibitors of pathological reactive astrocyte formation. a, Top results of HOMER transcription factor motif mining beneath pathological reactive astrocyte super-enhancers. b, Gene ontology analysis showing KEGG pathways enriched in genes associated with gained pathological reactive astrocyte super-enhancers. c, Representative western blot image of nuclear protein extracts probed for RelA/p65 and $\beta$-Actin. d, Quantification of experiments represented in $\mathrm{m}$. Data are presented as mean \pm s.e.m for an $n$ $=3$ biological replicates (independent astrocyte isolations). $p$-values generated by a one-way ANOVA with Dunnett post-test for multiple comparisons to reactive plus vehicle control. e, Full uncropped western blots that correspond to Fig. $2 \mathrm{~m}$. f, Distribution of NFkB inhibitors in the full primary screen drug library versus validated hits, showing that NFkB inhibitors are significantly enriched in the validated hit list. p-value generated by a hypergeometric test. $\mathbf{g}$, Heatmap showing the normalized NFkB luciferase activity in Jurkat reporter cells treated with the validated hits from the primary drug screen. Data presented as percentage of NFkB activity from a single biological replicate (astrocyte isolation) across an 8-point dose curve from 6.25uM to $0.1 \mathrm{uM}$. 


\section{Figure 3}
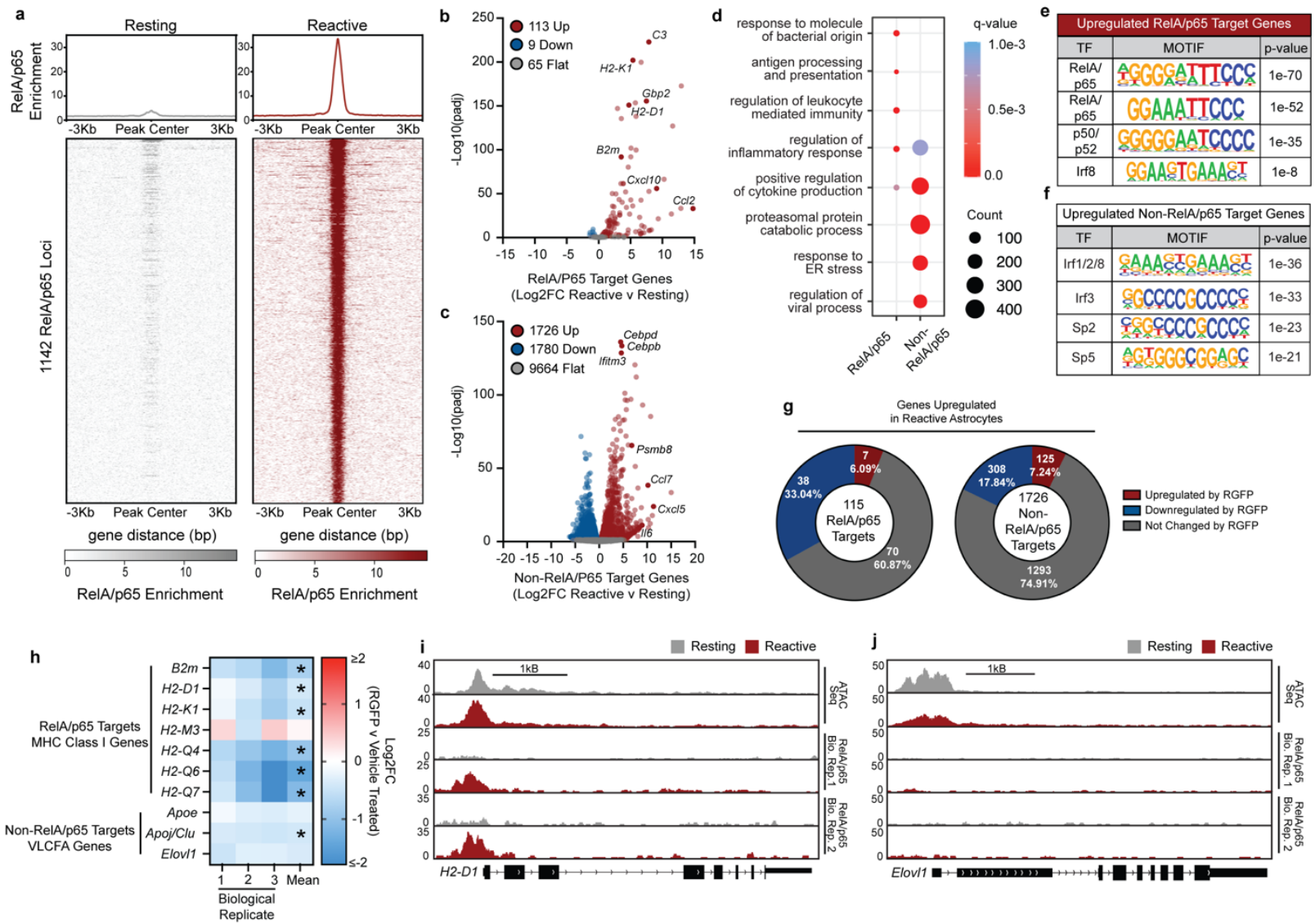

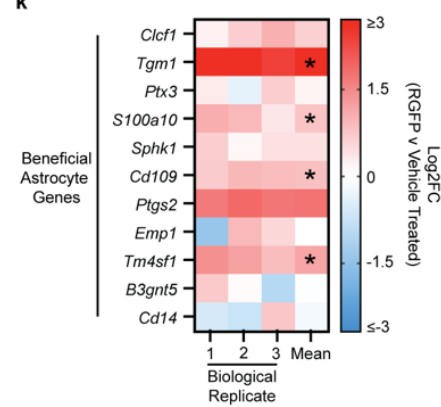

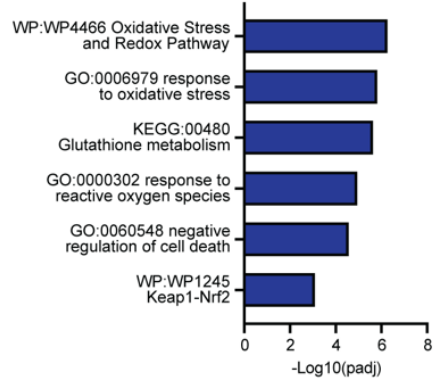

m

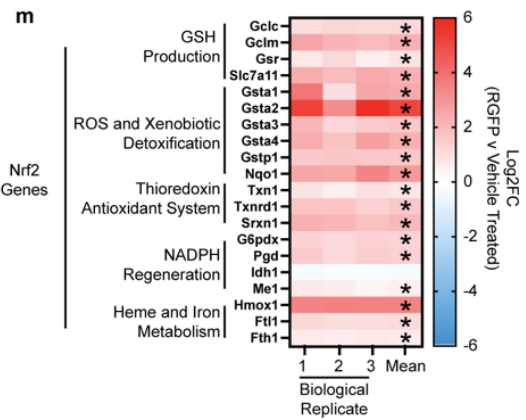

Figure 3. HDAC3 mediates a switch between pro-inflammatory and anti-inflammatory gene expression. a, RelA/p65 DNA binding aggregate heatmap and profile showing enriched RelA/p65 DNA binding at in reactive astrocytes compared to resting astrocytes. b, Volcano plot of RelA/p65 target genes (genes with a RelA/p65 peak within 5Kb up or downstream of the TSS). Of the 187 total RelA/p65 target genes in reactive astrocytes $60.43 \%$ (113/187) are upregulated while $4.81 \%$ $(9 / 187)$ are downregulated and $34.76 \%(65 / 187)$ do not change. Log2FC and padj values were generated from bulk RNAseq analysis with DESEQ2 and with an $n=3$ biological replicates (independent astrocyte isolations) per group. c, Volcano plot of the remaining Non-RelA/p65 target genes (genes without a RelA/p65 peak within $5 \mathrm{~Kb}$ up or downstream of the transcription start site). Of the 14,364 Non-RelA/p65 target genes $13.10 \%(1726 / 13170)$ are upregulated in reactive astrocytes while $13.51 \%(1780 / 13170)$ are downregulated and $73.39 \%(9664 / 13170)$ do 
not change. d, Comparison of gene ontology terms called for RelA/p65 and Non-RelA/p65 target genes that are significantly upregulated in pathological reactive astrocytes. e, Transcription factor motifs identified with ATAC-seq at the promoter of the ReIA/p65 target genes that are upregulated in reactive astrocytes compared to resting. $\mathbf{f}$, Transcription factor motifs identified with ATAC-seq at the promoter of the Non-RelA/p65 target genes that are upregulated in reactive astrocytes compared to resting. g, Distribution of the effect that treatment with the HDAC3 specific inhibitor RGFP966 (RGFP) has on RelA/p65 and Non-RelA/p65 target genes that are upregulated in reactive compared to resting astrocytes. $\mathbf{h}$, Heatmap showing the expression of the RelA/p65 MHC Class I genes and the non-RelA/p65 genes involved in very long-chain fatty acid synthesis and transport. Expression is shown as the Log2 fold-change (Log2FC) between 5uM RGFP966 and vehicle (DMSO) treated cells. Asterisks denote significance as called by DESeq2. i, Example browser track of the RelA/p65 target gene H2-D1 from the MHC Class I gene set. j, Example browser track of the non-RelA/p65 target gene Elovl1 that catalyzes the first and rate-limiting step in very long-chain fatty acid elongation. $\mathbf{k}$, Heatmap showing the difference in expression between 5uM RGFP and vehicle-treated pathological reactive astrocytes for genes associated with a beneficial reactive astrocyte. Expression is shown as Log2FC for RGFP966 versus vehicle with asterisks denoting significance as called by DESeq2. I, Gene ontology analysis of genes significantly increased by RGFP treatment. $\mathbf{m}$, Heatmap showing the difference in expression between 5uM RGFP and vehicle-treated pathological reactive astrocytes for cytoprotective Nrf2 target genes. Expression is shown as Log2FC for RGFP966 versus vehicle with asterisks denoting significance as called by DESeq2. 


\section{Extended Data Figure 5}
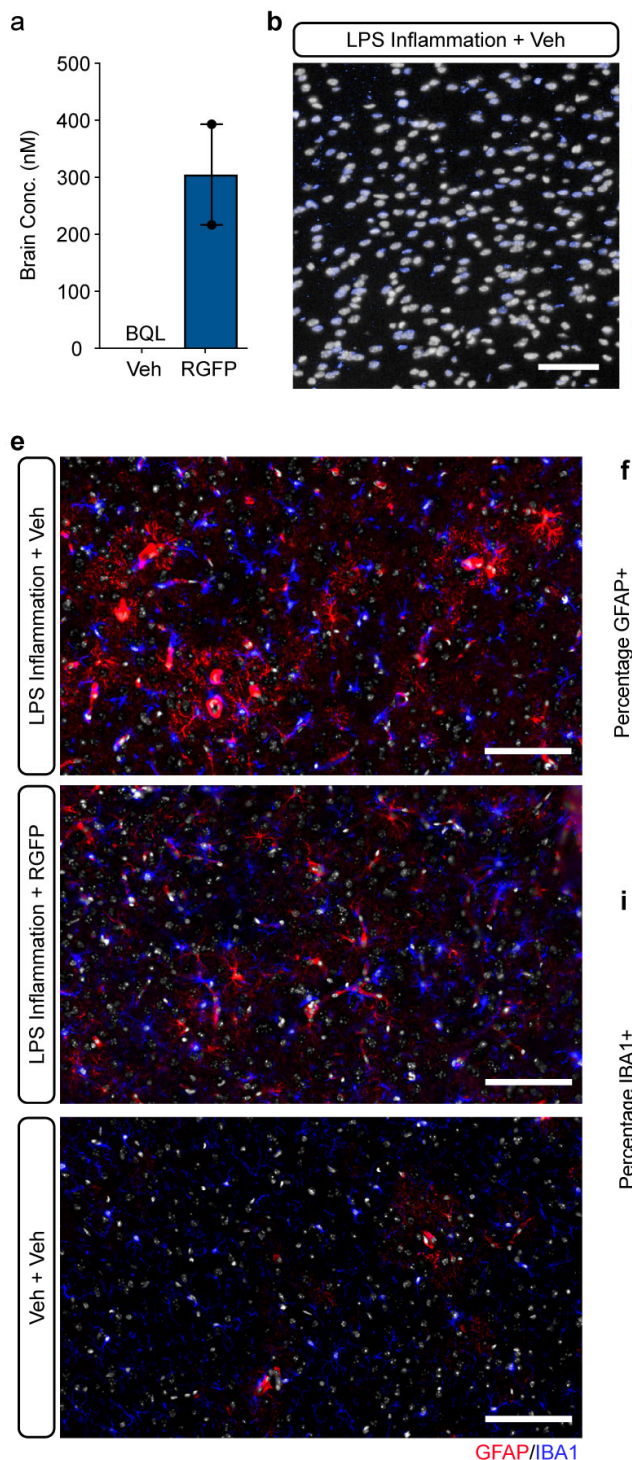
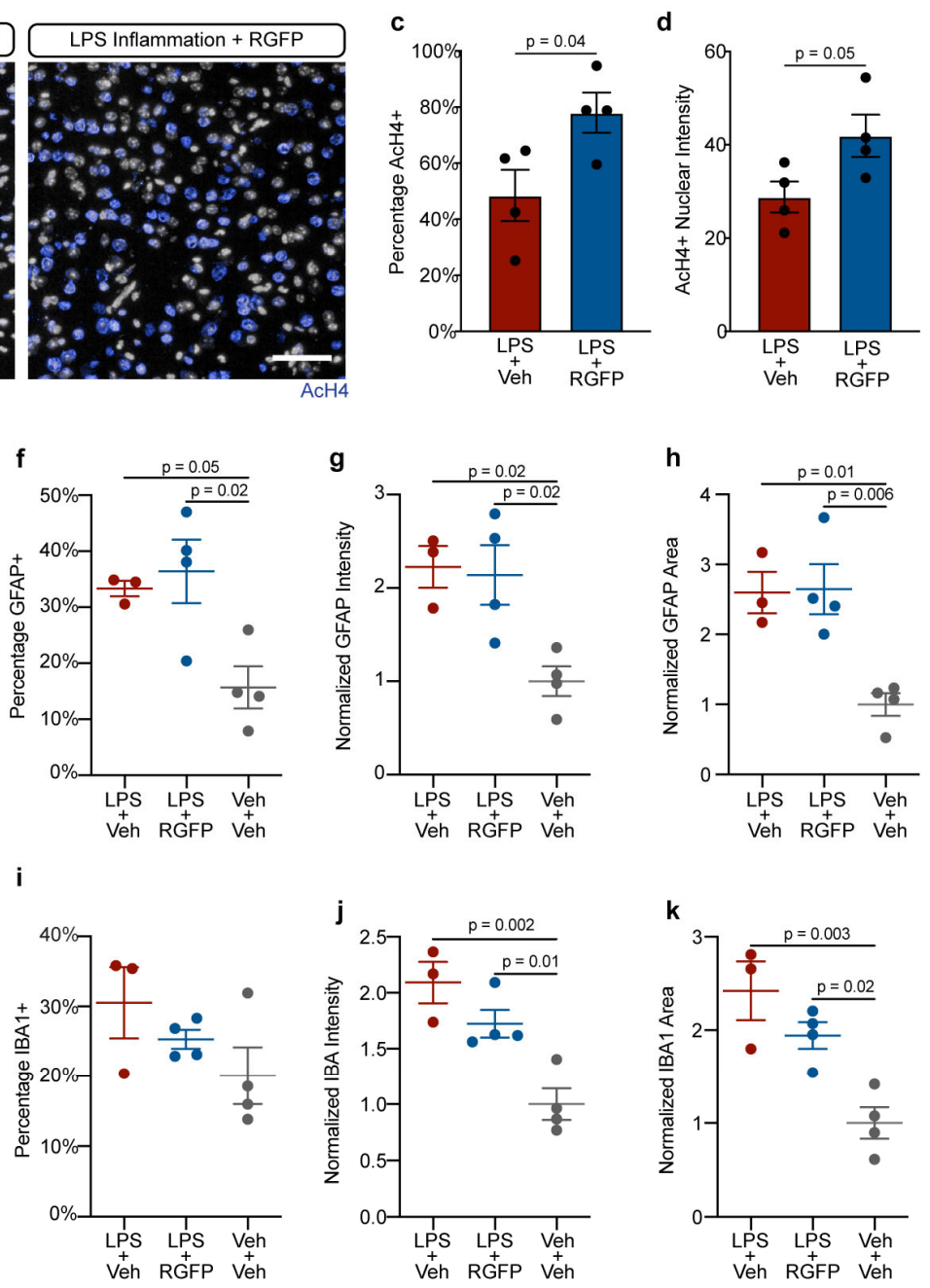

\section{Extended Data Figure 5. In vivo pharmacology of RGFP in mice challenged with systemic} LPS to induce neuroinflammation. a, Brain concentration of RGFP966 (RGFP) following treatment with vehicle (Veh) or $10 \mathrm{mg} / \mathrm{kg}$ RGFP. Data presented as mean \pm s.e.m. for $n=1-2$ biological replicates (mice). Concentration of RGFP in brain from vehicle treated mouse was below quantifiable levels (BQL). b, Representative images of immunohistochemistry for AcH4 (blue) in the cortex of mice treated chronically with vehicle or $10 \mathrm{mg} / \mathrm{kg}$ RGFP and then exposed to systemic LPS injections to induce neuroinflammation. Scale bar 100um. c, Quantification of the percentage of cells that are $\mathrm{AcH} 4$ positive from the experiment represented in $\mathrm{b}$. Data are presented as the mean \pm s.e.m. for $n=4$ biological replicates (mice) per group. $p$-value generated with a Student's two-tailed t-test. d, Quantification of AcH4 nuclear intensity from the experiment represented in b. Data are presented as the mean \pm s.e.m. for $n=4$ biological replicates (mice) per group. p-value generated with a Student's two-tailed t-test. e, Representative images of 
850 immunohistochemistry for GFAP (red) and IBA-1 (blue) in the cortex of mice treated with vehicle 851 or $10 \mathrm{mg} / \mathrm{kg}$ RGFP and exposed to systemic LPS or saline vehicle. Scale bar 100um. f-h, 852 Quantification of GFAP positive astrocytes in the experiment represented in e. Data are presented 853 for the $\mathbf{f}$, percentage of GFAP positive cells, $\mathbf{g}$, control-normalized GFAP intensity, and $\mathbf{h}$, control 854 normalized GFAP area. Data are presented as mean \pm s.e.m. for $n=3-4$ biological replicates 855 (mice) per group. p-value generated by one-way ANOVA with Tukey's post-test. i-k, 856 Quantification of IBA-1 positive microglia in the experiment represented in e. Data are presented 857 for the $\mathbf{i}$, percentage of cells IBA-1 positive, $\mathbf{j}$, control normalized IBA-1 intensity, and $\mathbf{k}$, control 858 normalized IBA-1 area. Data are presented as mean \pm s.e.m. for $n=3-4$ biological replicates 859 (mice) per group. p-value generated by one-way ANOVA with Tukey's post-test. 


\section{Extended Data Figure 6}
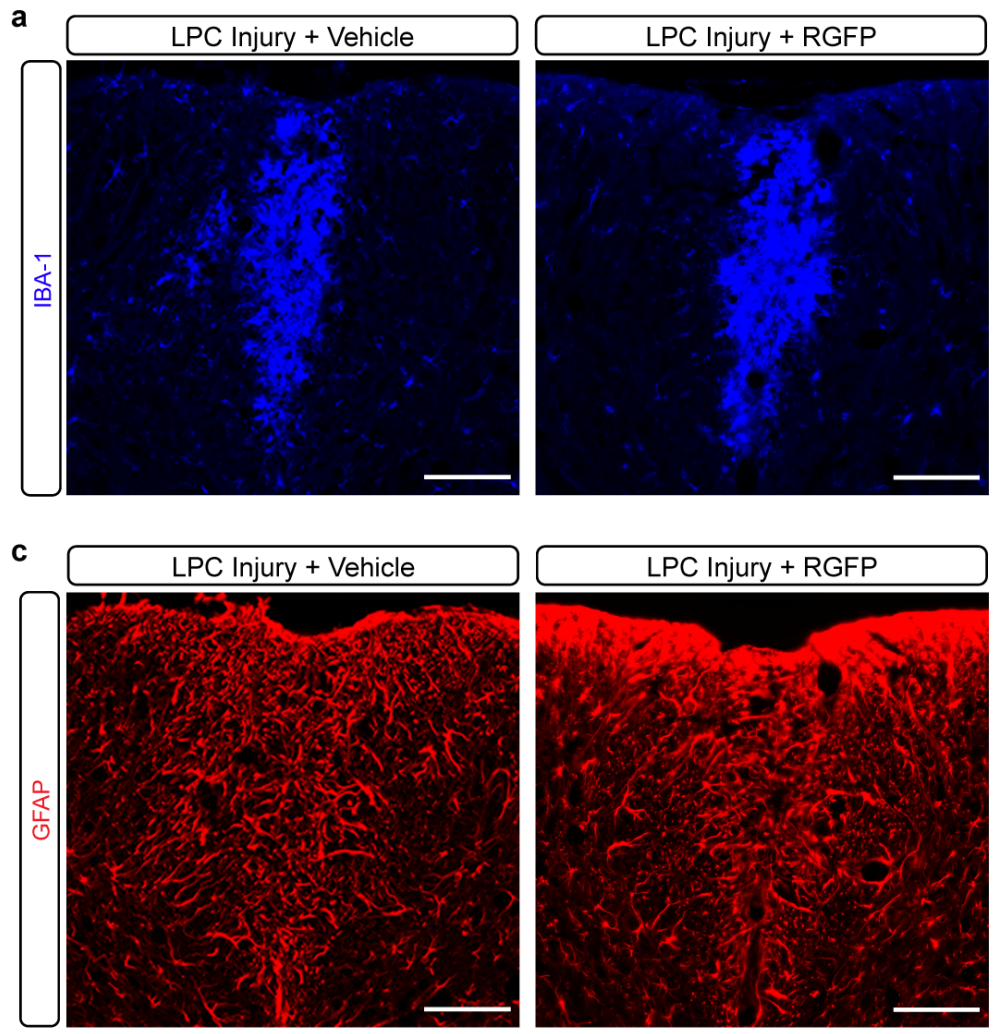

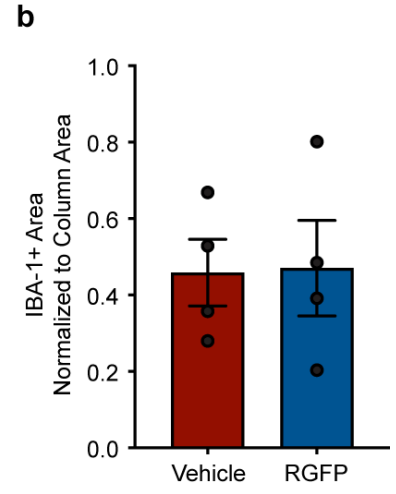

d

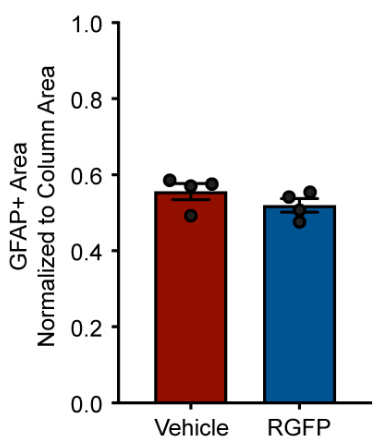

Extended Data Figure 6. RGFP966 has no effect on generalized gliosis in the toxin-based injury model LPC. a, Representative images of LPC lesions from vehicle or RGFP966 (RGFP) treated mice stained for IBA-1. Scale bar is $100 \mathrm{um}$. b, Quantification of the IBA-1 positive area divided by the dorsal column area from staining represented in a. Data are presented as mean \pm s.e.m. for $n=4$ biological replicates (mice) for each group. c, Representative images of LPC lesion from vehicle or RGFP treated mice stained for GFAP. Scale bar is 100um. d, Quantification of the GFAP-positive area divided by the dorsal column area from staining represented in a. Data are presented as mean \pm s.e.m. for $n=4$ biological replicates (mice) per group. 

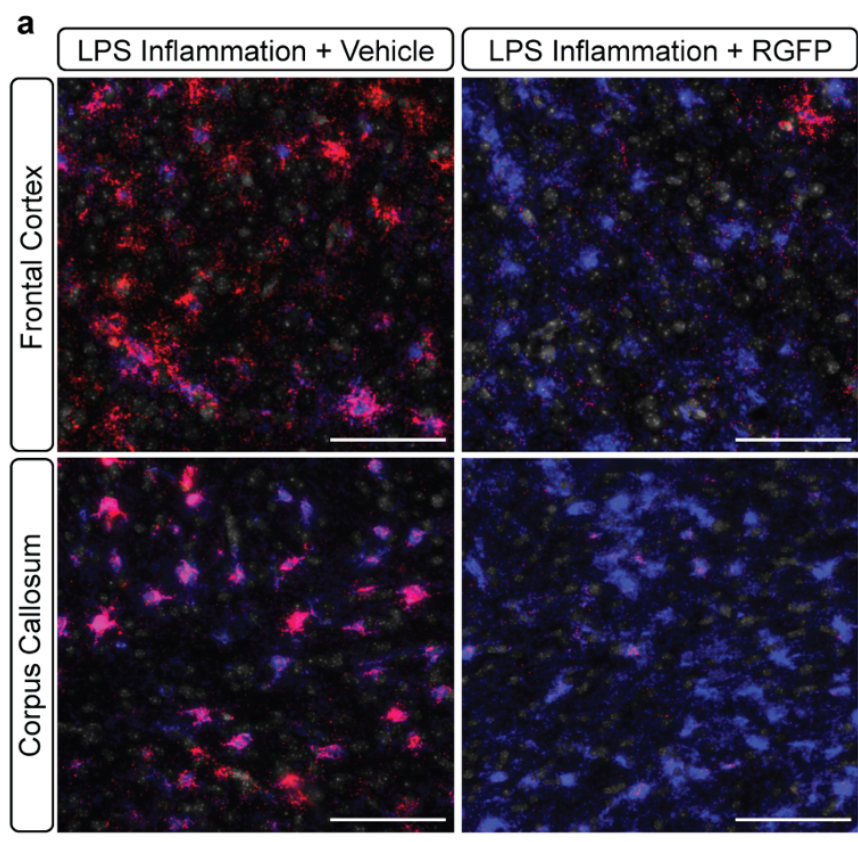

Gbp2/Gfap/DAPI
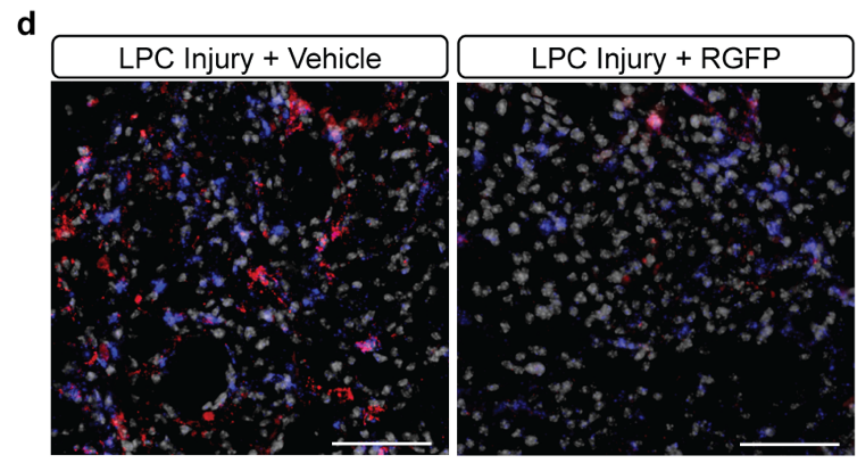

C3/SIc1a3/DAPI b
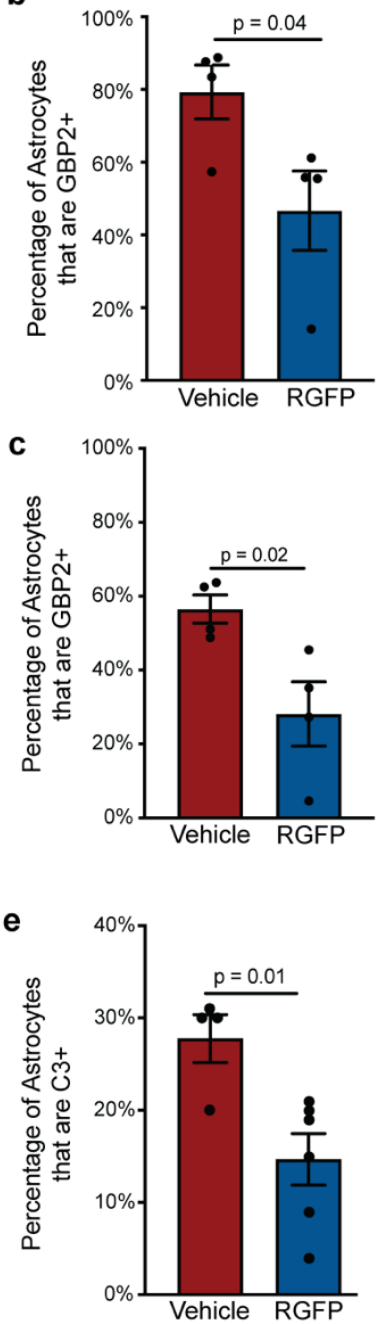
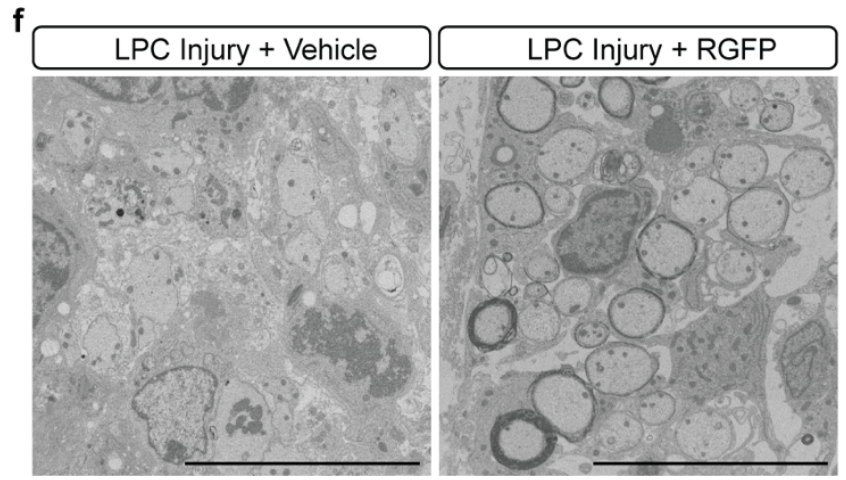

g

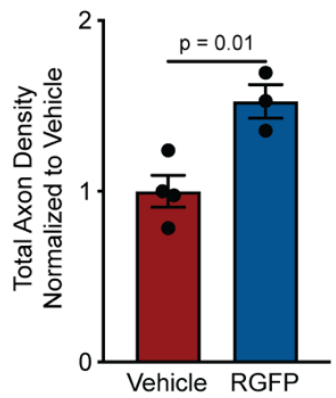

h

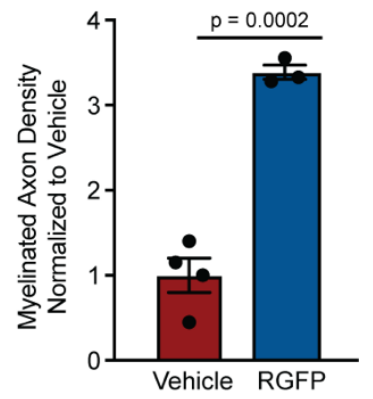

Figure. 4 HDAC3 inhibition blocks reactive astrocyte formation in vivo and promotes axonal protection. a) Representative images of Gbp2 (red) and Gfap (blue) in situ hybridization from the frontal cortex and corpus callosum of mice after 48hrs of systemic lipopolysaccharide (LPS) exposure and treated with vehicle or $10 \mathrm{mg} / \mathrm{kg}$ RGFP. Scale bar is $100 \mathrm{um}$. b-c, Quantification of in situ data represented in a. The percentage of astrocytes that are GBP2+ in 
the $\mathbf{b}$, frontal cortex or $\mathbf{c}$, corpus callosum of mice exposed to LPS and treated with vehicle or $10 \mathrm{mg} / \mathrm{kg}$ RGFP is presented as mean \pm s.e.m for $n=4$ biological replicates ( 2 male and 2 female mice) with p-value generated by Student's unpaired two-tailed t-test. $\mathbf{d}$, Representative images of C3 and S/c1a3 in situ hybridization in the dorsal column of lysolecithin (LPC) lesioned mice at 12 days post lesion and treated with vehicle or $10 \mathrm{mg} / \mathrm{kg}$ RGFP. Scale bar is $50 \mathrm{um}$. e, Quantification of in situ data represented in D. The percent of astrocytes that are C3 positive in LPC-exposed mice treated chronically with vehicle $10 \mathrm{mg} / \mathrm{kg}$ RGFP. Data are presented as the mean \pm s.e.m for $n=4-6$ biological replicates (mice) with $p$-value generated by Student's unpaired two-tailed t-test. f, Representative electron microscopy (EM) images of LPC lesioned mice treated with vehicle or $10 \mathrm{mg} / \mathrm{kg}$ RGFP. Scale bar is $10 \mathrm{um}$. g, Quantification of axon density in EM images represented in $\mathrm{f}$. Total axon density normalized to control for LPC lesioned mice treated with vehicle $(n=4$ mice) or $10 \mathrm{mg} / \mathrm{kg}$ RGFP ( $n=3$ mice) is presented as mean \pm s.e.m. with p-value generated by Student's unpaired two-tailed t-test. h) Quantification of remyelinated axon density in EM images represented in $\mathrm{f}$. Myelinated axon density normalized to control for LPC lesioned mice treated with vehicle or $10 \mathrm{mg} / \mathrm{kg}$ RGFP is presented as mean \pm s.e.m for $n=3-4$ biological replicates (mice) with $\mathrm{p}$-value generated by Student's unpaired two-tailed t-test. 